\title{
Test function method for blow-up phenomena of semilinear wave equations and their weakly coupled systems
}

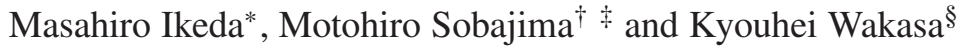

\begin{abstract}
In this paper we consider the wave equations with power type nonlinearities including timederivatives of unknown functions and their weakly coupled systems. We propose a framework of test function method and give a simple proof of the derivation of sharp upper bound of lifespan of solutions to nonlinear wave equations and their systems. We point out that for respective critical case, we use a family of self-similar solution to the standard wave equation including Gauss's hypergeometric functions which are originally introduced by Zhou [59]. However, our framework is much simpler than that. As a consequence, we found new $(p, q)$-curve for the system $\partial_{t}^{2} u-\Delta u=|v|^{q}, \partial_{t}^{2} v-\Delta v=\left|\partial_{t} u\right|^{p}$ and lifespan estimate for small solutions for new region.
\end{abstract}

Mathematics Subject Classification (2010): Primary: 35L05, 35L51, 35B44.

Key words and phrases: Semilinear wave equations, weakly coupled system, blowup, upper bound of lifespan, test function method

\section{Contents}

1 Introduction

2 Alternative proof of blowup of $\partial_{t}^{2} u-\Delta u=|u|^{p}$ for $1<p<p_{S}(N)$

3 Preliminaries for general cases 7

3.1 Super-solutions of wave equation and their properties $\ldots \ldots \ldots \ldots \ldots \ldots$

3.2 Self-similar solutions including Gauss's hyper geometric functions . . . . . . . . . . . 11

3.3 Lemmas for lifespan estimates for respective critical cases . . . . . . . . . . . . 13

4 The case $\partial_{t}^{2} u-\Delta u=G(u)$

5 The case $\partial_{t}^{2} u-\Delta u=G\left(\partial_{t} u\right)$

6 The case of a combined type $\partial_{t}^{2} u-\Delta u=G\left(u, \partial_{t} u\right)$

7 The case of the system $\partial_{t}^{2} u-\Delta u=G_{1}(v)$ and $\partial_{t}^{2} v-\Delta v=G_{2}(u)$

8 The case of the system $\partial_{t}^{2} u-\Delta u=G_{1}\left(\partial_{t} v\right)$ and $\partial_{t}^{2} v-\Delta v=G_{2}\left(\partial_{t} u\right)$

9 The case of system $\partial_{t}^{2} u-\Delta u=G_{1}(v)$ and $\partial_{t}^{2} v-\Delta v=G_{2}\left(\partial_{t} u\right)$

*Department of Mathematics, Faculty of Science and Technology, Keio University, 3-14-1 Hiyoshi, Kohoku-ku, Yokohama, 223-8522, Japan/Center for Advanced Intelligence Project, RIKEN, Japan, E-mail: masahiro.ikeda@keio.jp/masahiro.ikeda@riken.jp

$\dagger$ Department of Mathematics, Faculty of Science and Technology, Tokyo University of Science, 2641 Yamazaki, Noda-shi, Chiba, 278-8510, Japan, E-mail: msobajima1984@gmail.com

${ }^{\ddagger}$ Partially supported by Grant-in-Aid for Young Scientists Research (B) No.18K13445.

$\S$ Department of Mathematics, Faculty of Science and Technology, Tokyo University of Science, 2641 Yamazaki, Noda-shi, Chiba, 278-8510, Japan, E-mail: wakasa_kyouhei@ma.noda.tus . ac. jp 


\section{Introduction}

In this paper we consider the semilinear wave equations with power type nonlinearities including derivatives of unknown functions and their weakly coupled systems

$$
\begin{cases}\partial_{t}^{2} u(x, t)-\Delta u(x, t)=G\left(u(x, t), \partial_{t} u(x, t)\right), & (x, t) \in \mathbb{R}^{N} \times(0, T), \\ u(x, 0)=\varepsilon f(x) & x \in \mathbb{R}^{N}, \\ \partial_{t} u(x, 0)=\varepsilon g(x) & x \in \mathbb{R}^{N} .\end{cases}
$$

and

$$
\begin{cases}\partial_{t}^{2} u(x, t)-\Delta u(x, t)=G_{1}\left(v(x, t), \partial_{t} v(x, t)\right), & (x, t) \in \mathbb{R}^{N} \times(0, T), \\ \partial_{t}^{2} v(x, t)-\Delta v(x, t)=G_{2}\left(u(x, t), \partial_{t} u(x, t)\right), & (x, t) \in \mathbb{R}^{N} \times(0, T), \\ u(x, 0)=\varepsilon f_{1}(x), \quad v(x, 0)=\varepsilon f_{2}(x) & x \in \mathbb{R}^{N}, \\ \partial_{t} u(x, 0)=\varepsilon g_{1}(x), \quad \partial_{t} v(x, 0)=\varepsilon g_{2}(x) & x \in \mathbb{R}^{N},\end{cases}
$$

where $\partial_{t}=\partial / \partial t, \Delta=\sum_{j=1}^{N} \partial^{2} / \partial x_{j}^{2}$ and $T>0$. The nonlinear terms $G, G_{1}$ and $G_{2}$ are nonnegative and smooth (specified later) and $u$ and $v$ are unknown functions. Throughout this paper the initial values $(f, g),\left(f_{1}, g_{1}\right)$ and $\left(f_{2}, g_{2}\right)$ are assumed to be satisfied the following condition

$$
(f, g) \in C_{c}^{\infty}\left(\mathbb{R}^{N}\right), \quad I[g]:=\int_{\mathbb{R}^{N}} g(x) d x>0 .
$$

Finally, the parameter $\varepsilon>0$ describes the smallness of corresponding initial value. The aim of the present paper is to give a simple way to derive the corresponding sharp lifespan of blowup solutions to (1.1) and (1.2) via a test function method.

The problem of blowup phenomena of (1.1) has a long history. The study of this kind problem with $G(u)=|u|^{p}$ has been started by John [26]. He proved the following fact when $N=3$.

- If $1<p<1+\sqrt{2}$, then the solution of (1.1) blows up in finite time for "positive" initial value.

- If $p>1+\sqrt{2}$, then there exists a global solution with small initial value.

After that Strauss [51] conjectured that the threshold for dividing blowup phenomena in finite time for arbitrary "positive" small initial value and global existence of small solutions is given by

$$
p_{S}(N)=\sup \left\{p>1 ; \gamma_{S}(N, p)>0\right\}, \quad \gamma_{S}(N, p)=2+(N+1) p-(N-1) p^{2} .
$$

Actucally, this gives $p_{S}(3)=1+\sqrt{2}$ as mentioned above and in the case $N=1$, Kato [29] proved blowup phenomena in finite time for arbitrary "positive" small initial value with $1<p<p_{S}(1)=\infty$. There are many subsequent papers dealing with the blowup phenomena. Then until the contributions of Yordanov-Zhang [58] and Zhou [63], the complete picture of blowup phenomena and existence of global solutions are clarified including the critical situation $p=p_{S}(N)$ (see also Glassey [13, 14], Sideris [50], Schaeffer [47], Rammaha [46], Georgiev-Lindblad-Sogge [11]).

The lifespan of blowup solutions to (1.1) has been intensively considered. Here we refer Lindblad [40], Zhou [59, 60, 61], Lindblad-Sogge [41], Di Pomponio-Georgiev [8], Takamura-Wakasa [54] and Lai-Zhou [36]. In view of the previous works listed above, the precise behavior of lifespan of small solutions with respect to the parameter $\varepsilon>0$ sufficiently small:

$$
\operatorname{LifeSpan}(u) \approx \begin{cases}C \varepsilon^{-\frac{2 p(p-1)}{\gamma_{S}(N, p)}} & \text { if } 1<p<p_{S}(N), \\ \exp \left(C \varepsilon^{-p(p-1)}\right) & \text { if } p=p_{S}(N)\end{cases}
$$


An alternative proof of lifespan estimate with critical case $p=p_{S}(N)$ via Gauss's hypergeometric function can be found in Zhou [61] and Zhou-Han [65].

Similar problem can be found for (1.1) with $G=\left|\partial_{t} u\right|^{p}$ (see e.g., John [27], Sideris [49], Masuda [43], Schaeffer [48] Rammaha [45], Agemi [1] , Hidano-Tsutaya [17] Tzvetkov [55], Zhou [62] and Hidano-Wang-Yokoyama [18]). The complete picture of the blowup phenomena for small solutions can be summarized as follows:

$$
\operatorname{LifeSpan}(u) \approx \begin{cases}C \varepsilon^{-\left(\frac{1}{p-1}-\frac{N-1}{2}\right)^{-1}} & \text { if } 1<p<\frac{N+1}{N-1}, \\ \exp \left(C \varepsilon^{-(p-1)}\right) & \text { if } p=\frac{N+1}{N-1} . \\ \infty & \text { if } \frac{N+1}{N-1}<p<\frac{N}{N-2} .\end{cases}
$$

We should remark that the global existence of small solutions to 1.1) with $G=\left|\partial_{t} u\right|^{p}$ is only proved under the initial value with radially symmetric in high spatial dimension.

The problem (1.1) with combined type $G=|u|^{q}+\left|\partial_{t} u\right|^{p}$ has been recently discussed by Zhou-Han [64] and Hidano-Wang-Yokoyama [19]. In [19], it is found the borderline of the position of $(p, q)$ for blowup phenomena of small solutions. Surprisingly, in the threshold case they proved the global existence of small solutions which is completely different from the situation of both the cases $G(u)=|u|^{p}$ and $G=\left|\partial_{t} u\right|^{p}$.

In this connection, a similar interesting structure has been analysed for the weakly coupled problem (1.2). In this case the interaction of each unknown functions $u$ and $v$ plays an important role. In particular, the situation depends heavily on the structure of nonlinear terms $G_{1}$ and $G_{2}$. This means that even in the special case $G_{1}=|v|^{p}$ and $G_{2}=|u|^{q}$, the position of $(p, q)$ (with describes the effect of nonlinearity) is quite important to discuss the behavior of solutions to this system. From this view point, many mathematicians try to find the blowup phenomena and global solutions of small solutions. Here we refer Del Santo-Georgiev-Mitidieri [4], Deng [6], Del Santo-Mitidieri [5], Deng [7], Kubo-Ohta [31], AgemiKurokawa-Takamura [2], Kurokawa-Takamura [34], Kurokawa [33], Georgiev-Takamura-Zhou [12], Kurokawa-Takamura-Wakasa [35] for the case $G_{1}=|v|^{p}$ and $G_{2}=|u|^{q}$, Deng [7], Xu [57], KuboKubota-Sunagawa [32] for the case $G_{1}=\left|\partial_{t} v\right|^{p}$ and $G_{2}=\left|\partial_{t} u\right|^{q}$, and Hidano-Yokoyama [20] for the case $G_{1}=|v|^{q}$ and $G_{2}=\left|\partial_{t} u\right|^{p}$.

Recently in Ikeda-Sobajima [24] an alternative test function method for nonlinear heat, Schrödinger, and damped wave equations has been introduced and the sharp upper bound of lifespan for respective equations are given. Of course each equation has a huge mount of previous works (see e.g., Fujita [10], Hayakawa [16], Sugitani [52], Kobayashi-Sirao-Tanaka [28], Li-Nee [38] for the heat equations, IkedaWakasugi [25], Fujiwara-Ozawa [15] for Schrödinger equations and Li-Zhou [39], Lin-Nishihara-Zhai [42], Ikeda-Ogawa [21] Lai-Zhou [37] for damped wave equations the references therein). Despite of this, the technique in [24] gives us a short proof of sharp upper bound of lifespan of small solutions to respective equations and in some case, in particular the Schrödinger equation, the estimates given by this technique is not known. Moreover, it worth noticing that the initial value does not need to be required the positivity in the point-wise sense even in high dimensional cases $N \geq 4$. Therefore we expect that by introducing the technique in [24] into the analysis of wave equations, one can give an alternative proof of sharp (for many cases) upper bound of lifespan of small solutions and the assumption on the initial value can be weaken.

The first purpose of the present paper is to propose a framework of test function method for nonlinear wave equation due to [24] and give precise lifespan estimates for problems (1.1) and (1.2) without the assumption of the positivity of initial value in the point-wise sense. The second is to find the new blowup region for the case (1.2) with $G_{1}=|v|^{q}$ and $G_{2}=\left|\partial_{t} u\right|^{p}$ and lifespan estimates for respective cases. 
Since in the present paper we focus our attention to the framework of test function method, we do not enter a discussion for existence of solutions to the respective problems. At this point, we refer Sideris [50], Kapitanskii [30], Hidano-Wang-Yokoyama [18], Georgiev-Takamura-Zhou [12], Kubo-KubotaSunagawa [32], Hidano-Yokoyama [20] and their references therein.

The present paper is organized as follows: In Section 2 , to explain our argument, we demonstrate the short derivation of the upper bound of lifespan for the special case $\partial_{t}^{2} u-\Delta u=|u|^{p}$ with $1<p<p_{S}(N)$. Section 3 is devoted to describe the properties of super-solutions to the wave equations and self-similar solutions to the linear wave equation including Gauss's hypergeometric functions introduced in Zhou [59]. Some useful lemmas indicating our test function method are stated and proved also in Section 3. The main results are stated at the beginning of each Sections 4, 5, 6, 7, 8 and 9 . More precisely, in Section 4, we discuss

$$
\partial_{t}^{2} u-\Delta u=|u|^{p} \quad \text { in } \mathbb{R}^{N} \times(0, T)
$$

for the critical case $p=p_{S}(N)$. Although the sharp lifespan estimate has been proved by TakamuraWakasa [54] and an alternative proof was given by Zhou-Han [65], we will give a (much) simpler proof. Then we discuss the equation

$$
\partial_{t}^{2} u-\Delta u=\left|\partial_{t} u\right|^{p} \quad \text { in } \mathbb{R}^{N} \times(0, T)
$$

in Section 5 , which is related to Glassey conjecture. The eqaution with a combined type nonlinearity

$$
\partial_{t}^{2} u-\Delta u=|u|^{q}+\left|\partial_{t} u\right|^{p} \quad \text { in } \mathbb{R}^{N} \times(0, T)
$$

will be dealt with in Section 6 . After that the weakly coupled systems

$$
\begin{cases}\partial_{t}^{2} u-\Delta u=a_{11}|v|^{p_{11}}+a_{12}\left|\partial_{t} v\right|^{p_{12}}, & \text { in } \mathbb{R}^{N} \times(0, T), \\ \partial_{t}^{2} v-\Delta v=a_{21}|u|^{p_{21}}+a_{22}\left|\partial_{t} u\right|^{p_{22}}, & \text { in } \mathbb{R}^{N} \times(0, T),\end{cases}
$$

are considered when $a_{12}=a_{22}=0$ in Section7, when $a_{11}=a_{21}=0$ in Section 8 and when $a_{21}=a_{12}=0$ in Section 9 , respectively. We point out that in Section 9 a new blowup position of $(p, q)$ is found and lifespan estimates including critical situations (on the critical curve) are derived.

\section{Alternative proof of blowup of $\partial_{t}^{2} u-\Delta u=|u|^{p}$ for $1<p<p_{S}(N)$}

To begin with, we consider the following problem

$$
\begin{cases}\partial_{t}^{2} u_{\varepsilon}-\Delta u_{\varepsilon}=\left|u_{\varepsilon}\right|^{p} & \text { in } \mathbb{R}^{N} \times(0, T), \\ u_{\varepsilon}(0)=\varepsilon f & \text { in } \mathbb{R}^{N}, \\ \partial_{t} u_{\varepsilon}(0)=\varepsilon g & \text { in } \mathbb{R}^{N},\end{cases}
$$

where we assume that $f$ and $g$ satisfies (1.3). In this section we use

$$
\gamma_{S}(N, p)=2+(N+1) p-(N-1) p^{2}, \quad p_{S}(N)=\sup \left\{p>1 ; \gamma_{S}(N, p)>0\right\} .
$$

Definition 2.1. Let $f, g \in C_{c}^{\infty}\left(\mathbb{R}^{N}\right)$ and $p>1$. The function

$$
u \in C\left([0, T) ; H^{1}\left(\mathbb{R}^{N}\right)\right) \cap C^{1}\left([0, T) ; L^{2}\left(\mathbb{R}^{N}\right)\right) \cap L^{p}\left(0, T ; L^{p}\left(\mathbb{R}^{N}\right)\right)
$$


is called a weak solution of 2.1) in $(0, T)$ if $u(0)=\varepsilon f, \partial_{t} u(0)=\varepsilon g$ and for every $\Psi \in C_{c}^{\infty}\left(\mathbb{R}^{N} \times[0, T)\right.$,

$$
\begin{aligned}
& \varepsilon \int_{\mathbb{R}^{N}} g(x) \Psi(x, 0) d x+\int_{0}^{T} \int_{\mathbb{R}^{N}}|u(x, t)|^{p} \Psi(x, t) d x d t \\
& =\int_{0}^{T} \int_{\mathbb{R}^{N}}\left(-\partial_{t} u(x, t) \partial_{t} \Psi(x, t)+\nabla u(x, t) \cdot \nabla \Psi(x, t)\right) d x d t .
\end{aligned}
$$

Proposition 2.1. Let $f$, $g$ satisfy (1.3) and let $u_{\varepsilon}$ be a weak solution to (2.1) satisfying supp $u_{\varepsilon} \subset\{(x, t) \in$ $\left.\mathbb{R}^{N} \times[0, T] ;|x| \leq r_{0}+t\right\}$ for $r_{0}=\sup \{|x| ; x \in \operatorname{supp}(f, g)\}$. Set $T_{\varepsilon}$ as a lifespan of $u_{\varepsilon}$ given by

$$
T_{\varepsilon}=\sup \{T>0 ; \text { there exists a solution to } 2.10 \text { in }(0, T)\} \text {. }
$$

If $1<p<p_{S}(N)$ (that is, $\gamma_{S}(N, p) \geq 0$ ), then $T_{\varepsilon}<\infty$. Moreover, there exist $\varepsilon_{0}>0$ and $C>0$ such that for every $\varepsilon \in\left(0, \varepsilon_{0}\right]$,

$$
T_{\varepsilon} \leq \begin{cases}C \varepsilon^{-\frac{p-1}{2}} & \text { if } N=1,1<p<\infty, \\ C \varepsilon^{-\frac{p-1}{3-p}} & \text { if } N=2,1<p \leq 2, \\ C \varepsilon^{-2 p(p-1) / \gamma_{S}(2, p)} & \text { if } N=2,2<p<p_{S}(2), \\ C \varepsilon^{-2 p(p-1) / \gamma_{S}(N, p)} & \text { if } N \geq 3,1<p<p_{S}(N) .\end{cases}
$$

Proof. If $T_{\varepsilon} \leq 1$, then the assertion is trivial by choosing $\varepsilon_{0}$ sufficiently small. Suppose that $T_{\varepsilon}>1$ and take $T \in\left(1, T_{\varepsilon}\right)$. Put $\eta \in C^{\infty}([0, \infty))$ satisfying

$$
\eta(s)= \begin{cases}1 & s<1 / 2 \\ \text { decreasing } & 1 / 2<s<1, \quad \eta_{T}(s)=\eta(s / T) . \\ 0 & s>1,\end{cases}
$$

By the definition of weak solution of $(2.1)$ in $(0, T)$, we see from $\Psi=\eta_{T}(t)^{2 p^{\prime}}$ (multiplying compactly supported smooth function $\chi$ on $\mathbb{R}^{N}$ satisfying $\chi=1$ if $x \in B\left(0, r_{0}+T\right)$ )

$$
\begin{aligned}
I[g] \varepsilon+\int_{0}^{T} \eta_{T}^{2 p^{\prime}} \int_{\mathbb{R}^{N}}\left|u_{\varepsilon}\right|^{p} d x d t & =\int_{0}^{T} \int_{\mathbb{R}^{N}}\left(-\partial_{t} u_{\varepsilon} \partial_{t}\left(\eta_{T}^{2 p^{\prime}}\right)+\nabla u_{\varepsilon} \cdot \nabla\left(\eta_{T}^{2 p^{\prime}}\right)\right) d x d t \\
& =\int_{0}^{T} \int_{\mathbb{R}^{N}} u_{\varepsilon} \partial_{t}^{2}\left(\eta_{T}^{2 p^{\prime}}\right) d x d t \\
& =\frac{2 p^{\prime}}{T^{2}} \int_{0}^{T} \eta_{T}^{2 p^{\prime}-2} \int_{\mathbb{R}^{N}} u_{\varepsilon}\left(\eta(t / T) \eta^{\prime \prime}(t / T)+\left(2 p^{\prime}-1\right)\left(\eta^{\prime}(t / T)\right)^{2}\right) d x d t \\
& \leq \frac{2 p^{\prime}\left(\left\|\eta^{\prime \prime}\right\|_{L^{\infty}}+\left\|\eta^{\prime}\right\|_{L^{\infty}}^{2}\right)}{T^{2}} \int_{0}^{T} \eta_{T}^{2 p^{\prime} / p} \int_{\mathbb{R}^{N}}\left|u_{\varepsilon}\right| d x d t \\
& \leq \frac{\left[2 p^{\prime}\left(\left\|\eta^{\prime \prime}\right\|_{L^{\infty}}+\left\|\eta^{\prime}\right\|_{L^{\infty}}^{2}\right)\right]^{p^{\prime}}}{p^{\prime} T^{2 p^{\prime}}} \int_{0}^{T} \int_{B\left(r_{0}+t\right)} d x d t+\frac{1}{p} \int_{0}^{T} \eta_{T}^{2 p^{\prime}} \int_{\mathbb{R}^{N}}\left|u_{\varepsilon}\right|^{p} d x d t,
\end{aligned}
$$

where we have used the finite propagation property. This yields

$$
p^{\prime} I[g] \varepsilon+\int_{0}^{T} \eta_{T}^{2 p^{\prime}} \int_{\mathbb{R}^{N}}\left|u_{\varepsilon}\right|^{p} d x d t \leq C_{1} T^{N-1-\frac{2}{p^{-1}}}, \quad C_{1}:=\frac{\left[2 p^{\prime}\left(\left\|\eta^{\prime \prime}\right\|_{L^{\infty}}+\left\|\eta^{\prime}\right\|_{L^{\infty}}^{2}\right)\right]^{p^{\prime}}\left(1+r_{0}\right)^{N+1}\left|S^{N-1}\right|}{N(N+1)}
$$


with the volume of $N$-dimensional unit sphere $\left|S^{N-1}\right|$. Since the choice of $T \in\left(1, T_{\varepsilon}\right)$ is arbitrary, the above inequality implies the first and second estimates for $T_{\varepsilon}$.

To obtain the third and fourth estimates for $T_{\varepsilon}$, we introduce a special solution to linear wave equation as follows:

$$
w_{\lambda}(x, t)=\lambda^{N-1}\left((\lambda+t)^{2}-|x|^{2}\right)^{-\frac{N-1}{2}}, \quad \lambda>r_{0}
$$

Noting that $w_{\lambda}(x, 0) \rightarrow 1$ and $\partial_{t} w_{\lambda}(x, 0) \rightarrow 0$ as $\lambda \rightarrow \infty$ uniformly on $\operatorname{supp}(f, g)$, we see from dominated convergence theorem that there exists $\lambda_{0}>r_{0}$ such that

$$
\int_{\mathbb{R}^{N}} g(x) w_{\lambda_{0}}(x, 0)-f(x) \partial_{t} w_{\lambda_{0}}(x, 0) d x \geq \frac{1}{2} \int_{\mathbb{R}^{N}} g(x) d x=\frac{1}{2} I[g]>0 .
$$

Taking $\Psi=w_{\lambda_{0}} \eta_{T}^{2 p^{\prime}}$ (multiplying compactly supported smooth function $\chi$ on $\mathbb{R}^{N}$ satisfying $\chi=1$ if $\left.x \in B\left(0, r_{0}+T\right)\right)$ in the definition of weak solutions, we have

$$
\begin{aligned}
\varepsilon \int_{\mathbb{R}^{N}} g(x) w_{\lambda_{0}}(x, 0) d x+\int_{0}^{T} \int_{\mathbb{R}^{N}}\left|u_{\varepsilon}\right|^{p} \Psi d x d t & =\int_{0}^{T} \int_{\mathbb{R}^{N}}\left(-\partial_{t} u_{\varepsilon} \partial_{t} \Psi+\nabla u_{\varepsilon} \cdot \nabla \Psi\right) d x d t \\
& =\int_{\mathbb{R}^{N}} f(x) \partial_{t} w_{\lambda_{0}}(x, 0) d x+\int_{0}^{T} \int_{\mathbb{R}^{N}} u_{\varepsilon}\left(\partial_{t}^{2} \Psi-\Delta \Psi\right) d x d t .
\end{aligned}
$$

Neglecting the second term in the left-hand side and using Hölder's inequality and the definition of $w_{\lambda_{0}}$, we deduce

$$
\begin{aligned}
I[g] \varepsilon & \leq 2 \int_{0}^{T} \int_{\mathbb{R}^{N}} u_{\varepsilon}\left(\partial_{t}^{2} \Psi-\Delta \Psi\right) d x d t \\
& =4 p^{\prime} \int_{0}^{T} \eta_{T}^{2 p^{\prime}-2} \int_{\mathbb{R}^{N}} u_{\varepsilon}\left(2 \partial_{t} w_{\lambda_{0}} \frac{\eta^{\prime}(t / T) \eta(t / T)}{T}+w_{\lambda_{0}} \frac{\eta^{\prime \prime}(t / T) \eta(t / T)+\left(2 p^{\prime}-1\right)\left(\eta^{\prime}(t / T)\right)^{2}}{T^{2}}\right) d x d t \\
& \leq C_{2} T^{-N-1} \int_{T / 2}^{T} \eta_{T}^{2 p^{\prime} / p} \int_{\mathbb{R}^{N}}\left|u_{\varepsilon}\right|\left(1-\frac{|x|^{2}}{\left(\lambda_{0}+t\right)^{2}}\right)^{-\frac{N+1}{2}} d x d t \\
& \leq C_{2} T^{-N-1}\left(\int_{T / 2}^{T} \eta_{T}^{2 p^{\prime}} \int_{\mathbb{R}^{N}}\left|u_{\varepsilon}\right|^{p} d x d t\right)^{\frac{1}{p}}\left(\int_{T / 2}^{T} \int_{B\left(0, r_{0}+t\right)}\left(1-\frac{|x|}{\lambda_{0}+t}\right)^{-\frac{N+1}{2} p^{\prime}} d x d t\right)^{\frac{1}{p^{\prime}}} \\
& \leq C_{2}^{\prime}\left(T^{-N+\frac{N-1}{2} p} \int_{0}^{T} \eta_{T}^{2 p^{\prime}} \int_{\mathbb{R}^{N}}\left|u_{\varepsilon}\right|^{p} d x d t\right)^{\frac{1}{p}} .
\end{aligned}
$$

for some $C_{2}>0$ and $C_{2}^{\prime}>0$. Therefore we have

$$
(I[g] \varepsilon)^{p} T^{N-\frac{N-1}{2} p} \leq C_{2}^{\prime} \int_{0}^{T} \eta_{T}^{2 p^{\prime}} \int_{\mathbb{R}^{N}}\left|u_{\varepsilon}\right|^{p} d x d t .
$$

Combining (2.2) and (2.3), we obtain

$$
(I[g] \varepsilon)^{p} T^{N-\frac{N-1}{2} p} \leq C_{1} C_{2}^{\prime} T^{N-1-\frac{2}{p-1}}
$$

which implies the third and fourth estimates for $T_{\varepsilon}$.

Remark 2.1. The upper bound of $T_{\varepsilon}$ is not sharp in the case $(N, p)=(2,2)$. Indeed, Lindblad [40] Takamura [53] proved the estimate $T_{\varepsilon} \leq C a(\varepsilon)$ with $a^{2} \varepsilon^{2} \log (1+a)=1$ by using a refined concentration estimate (similar to (3.5) ) which is deduced from pointwise estimates for solutions to linear wave equation. 
Remark 2.2. In Yordanov-Zhang [58] and the subsequent papers, to prove a lower bound for $\int_{\mathbb{R}^{N}}|u|^{p} d x$, a positive radially symmetric solution $e^{-t} \phi(x)$ of $\partial_{t}^{2} u-\Delta u=0$ with the function $\phi$ satisfying $\phi-\Delta \phi=0$ were used. However, their treatment requires the positivity of initial value in the point-wise sense, in particular for high spatial dimensional cases. In contrast, the proof of Proposition 2.1 only needs the positivity of $I[g]$ by virtue of a new choice of solution $w_{\lambda}$.

Remark 2.3. In the proof of Proposition 2.1 we do not use neither an auxiliary result for second order ordinary inequalities nor an iteration argument. The view-point from the proof of Proposition 2.1 may give us an easier understanding about blowup phenomena for sub-critical case.

\section{Preliminaries for general cases}

To analyse more general equations and systems, we introduce the super-solutions to wave equations and self-similar solutions. In this section, we state the fundamental properties of the super-solutions to wave equations and self-similar solutions.

We note that even if some notations overlap with ones in the previous section, we state them again for the reader's convenience.

\subsection{Super-solutions of wave equation and their properties}

First we introduce super-solutions of wave equations.

Definition 3.1. Let $(f, g)$ satisfy (1.3). The function $u \in H^{1}\left(0, T ; L^{2}\left(\mathbb{R}^{N}\right)\right) \cap L^{2}\left(0, T ; H^{1}\left(\mathbb{R}^{N}\right)\right)$ is called a super-solution of $\partial_{t}^{2} u-\Delta u=H$ with $u(0)=\varepsilon f$ and $\partial_{t} u(0)=\varepsilon g$ and $H \in L^{1}\left(0, T ; L^{1}\left(\mathbb{R}^{N}\right)\right)$ if $u(0)=\varepsilon f$ and

$$
\varepsilon \int_{\mathbb{R}^{N}} g(x) \Psi(x, 0) d x+\int_{0}^{T} \int_{\mathbb{R}^{N}} H \Psi d x d t \leq \int_{0}^{T} \int_{\mathbb{R}^{N}}\left(-\partial_{t} u \partial_{t} \Psi+\nabla u \cdot \nabla \Psi\right) d x d t
$$

for every nonnegative function $\Psi \in C_{c}^{1}\left(\mathbb{R}^{N} \times[0, T)\right)$.

Then we will use two kinds of families of cut-off functions with respect to time variables; $\eta \in$ $C^{\infty}([0, \infty))$ satisfying

$$
\eta(s)= \begin{cases}1 & s<1 / 2 \\ \text { decreasing } & 1 / 2<s<1 \\ 0 & s>1\end{cases}
$$

and

$$
\eta^{*}(s)= \begin{cases}0 & s<1 / 2 \\ \eta(s) & s \geq 1 / 2\end{cases}
$$

and for $k \geq 2, R>0$,

$$
\eta_{R}(t)=\eta\left(\frac{t}{R}\right), \quad \eta_{R}^{*}(t)=\eta^{*}\left(\frac{t}{R}\right), \quad \psi_{R}(t)=\left[\eta_{R}(t)\right]^{k}, \quad \psi_{R}^{*}(t)=\left[\eta_{R}^{*}(t)\right]^{k} .
$$

The functions $\eta_{R}^{*}$ and $\psi_{R}^{*}$ are used only to justify the following estimates.

Lemma 3.1. Let $k \geq 2$ and $R \geq 1$. For every $t \geq 0$,

$$
\left|\partial_{t} \psi_{R}(t)\right| \leq \frac{k\left\|\eta^{\prime}\right\|_{L^{\infty}}}{R}\left[\psi_{R}^{*}(t)\right]^{1-\frac{1}{k}}, \quad\left|\partial_{t}^{2} \psi_{R}(t)\right| \leq \frac{k\left((k-1)\left\|\eta^{\prime}\right\|_{L^{\infty}}^{2}+\left\|\eta^{\prime \prime} \eta\right\|_{L^{\infty}}\right)}{R^{2}}\left[\psi_{R}^{*}(t)\right]^{1-\frac{2}{k}} .
$$


Proof. Noting that

$$
\begin{aligned}
\partial_{t} \psi_{R}(t) & =k \eta_{R}^{\prime}(t)\left[\eta_{R}(t)\right]^{k-1} \\
\partial_{t}^{2} \psi_{R}(t) & =k\left((k-1)\left(\eta_{R}^{\prime}(t)\right)^{2}+\eta_{R}(t) \eta_{R}^{\prime \prime}(t)\right)\left[\eta_{R}(t)\right]^{k-2},
\end{aligned}
$$

we easily obtain the desired inequalities.

By using $\psi_{R}$ as a test function for super-solutions, we obtain the following lemma.

Lemma 3.2. Let $1<p<\infty$ and $k \geq 2 p^{\prime}$, and let $(f, g)$ satisfy (1.3) and let $u$ be a super-solution of $\partial_{t}^{2} u-\Delta u=H$ with $u(0)=\varepsilon f, \partial_{t} u(0)=\varepsilon g, H \in L^{2}\left(0, T ; L^{2}\left(\mathbb{R}^{N}\right)\right)$ and $\operatorname{supp} u \subset\left\{(x, t) \in \mathbb{R}^{N} \times[0, T] ;|x| \leq\right.$ $\left.r_{0}+t\right\}$ for $r_{0}=\sup \{|x| ; x \in \operatorname{supp}(f, g)\}$. Then the following inequalities hold:

(i) For every $1 \leq R<T$

$$
I[g] \varepsilon+\int_{0}^{T} \int_{\mathbb{R}^{N}} H \psi_{R} d x d t \leq C_{1} R^{-2} \int_{0}^{T} \int_{\mathbb{R}^{N}}|u|\left[\psi_{R}^{*}\right]^{\frac{1}{p}} d x d t
$$

(ii) For every $1 \leq R<T$

$$
I[g] \varepsilon+\int_{0}^{T} \int_{\mathbb{R}^{N}} H \psi_{R} d x d t \leq C_{2} R^{-1} \int_{0}^{T} \int_{\mathbb{R}^{N}}\left|\partial_{t} u\right|\left[\psi_{R}^{*}\right]^{\frac{1}{p}} d x d t .
$$

Proof. By the definition of super-solution of $\partial_{t}^{2} u-\Delta u=H \geq 0$, choosing $\Psi=\psi_{R}$ (with multiplying compactly supported smooth function $\zeta$ satisfying $\zeta \equiv 1$ on supp $u$ ), we have

$$
I[g] \varepsilon+\int_{0}^{T} \int_{\mathbb{R}^{N}} H \psi_{R} d x d t \leq-\int_{0}^{T} \int_{\mathbb{R}^{N}} \partial_{t} u \partial_{t} \psi_{R} d x d t
$$

Then we can obtain (ii) by using Lemma 3.1 with $k \geq p^{\prime}$. On the other hand, noting that

$$
\int_{\mathbb{R}^{N}} \partial_{t} u \partial_{t} \psi_{R} d x=\frac{d}{d t} \int_{\mathbb{R}^{N}} u \partial_{t} \psi_{R} d x-\int_{\mathbb{R}^{N}} u \partial_{t}^{2} \psi_{R} d x
$$

we see from 3.1 with $k \geq 2 p^{\prime}$.

For general (smooth) test function $\Psi$, we can find the following relation with respect to $\partial_{t}^{2} \Psi-\Delta \Psi$.

Lemma 3.3. Let $u$ be a super-solution of $\partial_{t}^{2} u-\Delta u=H$ with $u(0)=\varepsilon f, \partial_{t} u(0)=\varepsilon g$ and $H \geq 0$. Then the following inequalities hold:

(i) For $\Psi \in C^{2}\left(\mathbb{R}^{N} \times[0, T)\right)$ satisfying $\Psi \geq 0$,

$$
\varepsilon \int_{\mathbb{R}^{N}}\left(g \Psi(\cdot, 0)-f \partial_{t} \Psi(\cdot, 0)\right) d x+\int_{0}^{T} \int_{\mathbb{R}^{N}} H \Psi d x d t \leq \int_{0}^{T} \int_{\mathbb{R}^{N}} u\left(\partial_{t}^{2} \Psi-\Delta \Psi\right) d x d t .
$$

(ii) For $\widetilde{\Psi} \in C^{3}\left(\mathbb{R}^{N} \times[0, T)\right)$ satisfying $\partial_{t} \widetilde{\Psi} \geq 0$,

$$
\varepsilon \int_{\mathbb{R}^{N}}\left(g \partial_{t} \widetilde{\Psi}(\cdot, 0)-f \Delta \widetilde{\Psi}(\cdot, 0)\right) d x+\int_{0}^{T} \int_{\mathbb{R}^{N}} H \partial_{t} \widetilde{\Psi} d x d t \leq \int_{0}^{T} \int_{\mathbb{R}^{N}} \partial_{t} u\left(\partial_{t}^{2} \widetilde{\Psi}-\Delta \widetilde{\Psi}\right) d x d t
$$


Proof. (i) Integration by parts yields that

$$
\begin{aligned}
\int_{0}^{T} \int_{\mathbb{R}^{N}}\left(-\partial_{t} u \partial_{t} \Psi+\nabla u \cdot \nabla \Psi\right) d x d t & =\frac{d}{d t}\left[-\int_{\mathbb{R}^{N}} u \partial_{t} \Psi d x\right]+\int_{0}^{T} \int_{\mathbb{R}^{N}}\left(u \partial_{t}^{2} \Psi-\Delta u \Psi\right) d x d t \\
& =\varepsilon \int_{\mathbb{R}^{N}} f \partial_{t} \Psi(x, 0) d x+\int_{0}^{T} \int_{\mathbb{R}^{N}} u\left(\partial_{t}^{2} \Psi-\Delta \Psi\right) d x d t .
\end{aligned}
$$

Connecting the definition of super-solution, we deduce (3.3).

(ii) Applying (i) $\Psi=\partial_{t} \widetilde{\Psi} \in C_{c}^{2}\left(\mathbb{R}^{N} \times[0, T)\right)$, we have

$$
\varepsilon \int_{\mathbb{R}^{N}}\left(g \partial_{t} \widetilde{\Psi}(\cdot, 0)-f \partial_{t}^{2} \widetilde{\Psi}(\cdot, 0)\right) d x+\int_{0}^{T} \int_{\mathbb{R}^{N}} H \widetilde{\Psi} d x d t \leq \int_{0}^{T} \int_{\mathbb{R}^{N}} u \partial_{t}\left(\partial_{t}^{2} \widetilde{\Psi}-\Delta \widetilde{\Psi}\right) d x d t .
$$

Noting that

$$
\begin{aligned}
\int_{0}^{T} \int_{\mathbb{R}^{N}} u \partial_{t}\left(\partial_{t}^{2} \widetilde{\Psi}-\Delta \widetilde{\Psi}\right) d x d t & =\int_{0}^{T} \frac{d}{d t}\left[\int_{\mathbb{R}^{N}} u\left(\partial_{t}^{2} \widetilde{\Psi}-\Delta \widetilde{\Psi}\right) d x\right] d t-\int_{0}^{T} \int_{\mathbb{R}^{N}} \partial_{t} u\left(\partial_{t}^{2} \widetilde{\Psi}-\Delta \widetilde{\Psi}\right) d x d t \\
& =-\varepsilon \int_{\mathbb{R}^{N}} f\left(\partial_{t}^{2} \widetilde{\Psi}(\cdot, 0)-\Delta \widetilde{\Psi}(\cdot, 0)\right) d x d t-\int_{0}^{T} \int_{\mathbb{R}^{N}} \partial_{t} u\left(\partial_{t}^{2} \widetilde{\Psi}-\Delta \widetilde{\Psi}\right) d x d t
\end{aligned}
$$

we have (3.4).

The following two lemmas describe the concentration phenomena of the wave near the light cone $\partial B(0,1+t)$, which is essentially the same as an estimate given in Yordanov-Zhang [58]. In their proofs, we use a special solution of linear wave equation given by

$$
V(x, t)=t\left(t^{2}-|x|^{2}\right)^{-\frac{N+1}{2}}=t^{-N}\left(1-\frac{|x|^{2}}{t^{2}}\right)^{-\frac{N+1}{2}}
$$

in $Q=\left\{(x, t) \in \mathbb{R}^{N} \times[0, \infty) ;|x|<t\right\}$. By the notation in next subsection, we see $V(x, t)=\Phi_{\beta}(x, t)$ with $\beta=N$ (see (3.2) below).

Lemma 3.4. Let $f, g$ satisfy (1.3) and let $u$ be a super-solution of $\partial_{t}^{2} u-\Delta u=0$ with $u(0)=\varepsilon f$, $\partial_{t} u(0)=\varepsilon g$ and $\operatorname{supp} u \subset\left\{(x, t) \in \mathbb{R}^{N} \times[0, T] ;|x| \leq r_{0}+t\right\}$ for $r_{0}=\sup \{|x| ; x \in \operatorname{supp}(f, g)\}$. Then for every $p>1$ and $k \geq 2 p^{\prime}$, there exists a constant $\delta_{1}=\delta_{1}(N, p, k, f, g)>0$ (independent of $\varepsilon$ ) such that for every $1 \leq R<T$

$$
\delta_{1}(I[g] \varepsilon)^{p} R^{N-\frac{N-1}{2} p} \leq \int_{0}^{T} \int_{\mathbb{R}^{N}}|u|^{p} \psi_{R}^{*} d x d t
$$

Proof. Put

$$
v_{\lambda}(x, t)=\lambda^{N} V(x, \lambda+t), \quad(x, t) \in Q_{\lambda}=\left\{(x, t) \in \mathbb{R}^{N} \times[0, \infty) ;(x, \lambda+t) \in Q\right\} .
$$

for $\lambda>r_{0}$ and then supp $u \subset Q_{\lambda}$. Noting that

$$
\partial_{t} V(x, t)=-t^{-N-1}\left(N+\frac{|x|^{2}}{t^{2}}\right)\left(1-\frac{|x|^{2}}{t^{2}}\right)^{-\frac{N+3}{2}},
$$


we see that

$$
\begin{aligned}
& \int_{\mathbb{R}^{N}} g(x) v_{\lambda}(x, 0)-f(x) \partial_{t} v_{\lambda}(x, 0) d x \\
& =\int_{\mathbb{R}^{N}} g(x)\left(1-\frac{|x|^{2}}{\lambda^{2}}\right)^{-\frac{N+1}{2}} d x+\frac{1}{\lambda} \int_{\mathbb{R}^{N}} f(x)\left(N+\frac{|x|^{2}}{\lambda^{2}}\right)\left(1-\frac{|x|^{2}}{\lambda^{2}}\right)^{-\frac{N+3}{2}} d x .
\end{aligned}
$$

Since the pair $(f, g)$ satisfies (1.3), the dominated convergence theorem implies that there exists $\lambda_{0}>r_{0}$ such that

$$
\int_{\mathbb{R}^{N}} g(x) v_{\lambda_{0}}(x, 0)-f(x) \partial_{t} v_{\lambda_{0}}(x, 0) d x \geq \frac{1}{2} \int_{\mathbb{R}^{N}} g(x) d x>0 .
$$

On the other hand, since $u$ is a super-solution of $\partial_{t}^{2} u-\Delta u=0$, choosing $\Psi=v_{\lambda_{0}} \psi_{R}$ in Lemma 3.3 (i), we see from the fact $\partial_{t}^{2} v_{\lambda_{0}}-\Delta v_{\lambda_{0}}=0$ and Lemma 3.1 that

$$
\begin{aligned}
& \varepsilon \int_{\mathbb{R}^{N}} g(x) v_{\lambda_{0}}(x, 0)-f(x) \partial_{t} v_{\lambda_{0}}(x, 0) d x \\
& \leq \int_{0}^{T} \int_{\mathbb{R}^{N}} u\left(\partial_{t}^{2}\left(v_{\lambda_{0}} \psi_{R}\right)-\Delta\left(v_{\lambda_{0}} \psi_{R}\right)\right) d x d t \\
& \leq C \int_{0}^{T} \int_{\mathbb{R}^{N}}|u|\left(\frac{\left|\partial_{t} v_{\lambda_{0}}\right|}{R}+\frac{v_{\lambda_{0}}}{R^{2}}\right)\left[\psi_{R}^{*}\right]^{\frac{1}{p}} d x d t \\
& \leq C\left(\int_{0}^{T} \int_{\mathbb{R}^{N}}|u|^{p} \psi_{R}^{*} d x d t\right)^{\frac{1}{p}}\left(\int_{\frac{R}{2}}^{R} \int_{B(0,1+t)}\left(\frac{\left|\partial_{t} v_{\lambda_{0}}\right|}{R}+\frac{v_{\lambda_{0}}}{R^{2}}\right)^{p^{\prime}} d x d t\right)^{\frac{1}{p^{\prime}}} .
\end{aligned}
$$

Since $|x| \leq 1+t$ and $R / 2 \leq t \leq R$ yield

$$
\frac{\left|\partial_{t} \nu_{\lambda_{0}}\right|}{R}+\frac{\nu_{\lambda_{0}}}{R^{2}} \leq C R^{-N-2}\left(1-\frac{|x|^{2}}{\left(\lambda_{0}+t\right)^{2}}\right)^{-\frac{N+3}{2}} \leq C R^{-N-2}\left(1-\frac{|x|}{\lambda_{0}+t}\right)^{-\frac{N+3}{2}},
$$

a direct calculation implies

$$
\int_{\frac{R}{2}}^{R} \int_{B(0,1+t)}\left(\frac{\left|\partial_{t} v_{\lambda_{0}}\right|}{R}+\frac{v_{\lambda_{0}}}{R^{2}}\right)^{p^{\prime}} d x d t \leq C R^{N-\left(\frac{N+1}{2}\right) p^{\prime}} .
$$

Combining (3.6), (3.7) and (3.8), we obtain (3.5).

Lemma 3.5. Let $f, g$ satisfy (1.3) and let $u$ be a super-solution of $\partial_{t}^{2} u-\Delta u=0$ with $u(0)=\varepsilon f$, $\partial_{t} u(0)=\varepsilon g$ and $\operatorname{supp} u \subset\left\{(x, t) \in \mathbb{R}^{N} \times[0, T] ;|x| \leq r_{0}+t\right\}$ for $r_{0}=\sup \{|x| ; x \in \operatorname{supp}(f, g)\}$. Then for every $q>1$ and $k \geq 2 q^{\prime}$, there exists a constant $\delta_{1}^{\prime}=\delta_{1}^{\prime}(N, q, k, f, g)>0$ such that for every $1 \leq R<T$

$$
\delta_{1}^{\prime}(I[g] \varepsilon)^{q} R^{N-\frac{N-1}{2} q} \leq \int_{0}^{T} \int_{\mathbb{R}^{N}}\left|\partial_{t} u\right|^{q} \psi_{R}^{*} d x d t
$$

Proof. Set $w_{\lambda}(x, t)=-\lambda^{N+1} V_{\lambda}(x, t)=-\lambda v_{\lambda}(x, t)$ for $\lambda>1$ and then

$$
\partial_{t}\left(w_{\lambda} \psi_{R}\right)=\lambda\left(-\partial_{t} v_{\lambda} \psi_{R}-v_{\lambda} \partial_{t} \psi_{R}\right) \geq 0
$$


Noting that $\partial_{t}^{2} w_{\lambda}-\Delta w_{\lambda}=0$, as in the proof of Lemma 3.4, we can verify that there exists $\lambda_{0}^{\prime}>1$ such that

$$
\begin{aligned}
\int_{\mathbb{R}^{N}} g(x) \partial_{t} w_{\lambda_{0}^{\prime}}(x, 0)-f(x) \Delta w_{\lambda_{0}^{\prime}}(x, 0) d x & =-\lambda \int_{\mathbb{R}^{N}} g(x) \partial_{t} v_{\lambda_{0}^{\prime}}(x, 0) d x+\lambda \int_{\mathbb{R}^{N}} f(x) \partial_{t}^{2} v_{\lambda_{0}^{\prime}}(x, 0) d x \\
& \geq \frac{N}{2} I[g] .
\end{aligned}
$$

Applying Lemma 3.3 (ii) with $\widetilde{\Psi}=w_{\lambda_{0}^{\prime}} \psi_{R}$, we have

$$
\begin{aligned}
\varepsilon \int_{\mathbb{R}^{N}} g(x) \partial_{t} v_{\lambda_{0}^{\prime}}(x, 0)-f(x) \Delta v_{\lambda_{0}^{\prime}}(x, 0) d x & \leq-\int_{0}^{T} \int_{\mathbb{R}^{N}} \partial_{t} u\left(\partial_{t}^{2}\left(w_{\lambda_{0}^{\prime}} \psi_{R}\right)-\Delta\left(w_{\lambda_{0}^{\prime}} \psi_{R}\right)\right) d x d t \\
& \leq C \int_{0}^{T} \int_{\mathbb{R}^{N}}\left|\partial_{t} u\right|\left(\frac{\left|\partial_{t} v_{\lambda_{0}^{\prime}}\right|}{R}+\frac{v_{\lambda_{0}^{\prime}}}{R^{2}}\right)\left[\psi_{R}^{*}\right]^{\frac{1}{p}} d x d t \\
& \leq C R^{\frac{N-1}{2}-\frac{N}{q}}\left(\int_{0}^{T} \int_{\mathbb{R}^{N}}\left|\partial_{t} u\right|^{q} \psi_{R}^{*} d x d t\right)^{\frac{1}{q}} .
\end{aligned}
$$

The above inequalities imply (3.9).

\subsection{Self-similar solutions including Gauss's hyper geometric functions}

In the respective critical case of blowup phenomena for wave equations, we need a precise information about the behavior of solutions to the linear wave equations. Therefore, next we introduce a family of self-similar solutions to $\partial_{t}^{2} u-\Delta u=0$ including Gauss's hypergeometric functions which also can be found in Zhou [59, 60, 61], Zhou-Han [65] and also Ikeda-Sobajima [22, 23].

Definition 3.2. Let $N \geq 2$. For $0<\beta<\infty$, define

$$
\Phi_{\beta}(x, t)=(t+|x|)^{-\beta} F\left(\beta, \frac{N-1}{2}, N-1 ; \frac{2|x|}{t+|x|}\right) \quad(x, t) \in Q,
$$

where $F(a, b, c ; z)$ is the Gauss hypergeometric function with a parameter $(a, b, c)$ given by

$$
F(a, b, c ; z)=\sum_{n=0}^{\infty} \frac{(a)_{n}(b)_{n}}{(c)_{n}} \frac{z^{n}}{n !}, \quad z \in[0,1)
$$

with the The Pochhammer symbol $(d)_{0}=1$ and $(d)_{n}=\prod_{k=1}^{n}(d+k-1)$ for $n \in \mathbb{N}$. Also we set

$$
\Phi_{\beta, \lambda}(x, t)=\lambda^{\beta} \Phi_{\beta}(x, \lambda+t), \quad(x, t) \in Q_{\lambda}=\left\{(x, t) \in \mathbb{R}^{N} \times[0, \infty) ;(x, \lambda+t) \in Q\right\} .
$$

Remark 3.1. In particular, the following formula for hypergeometric functions is known:

$$
F\left(a, a+\frac{1}{2}, c ; z\right)=(1+\sqrt{z})^{-2 a} F\left(2 a, c-\frac{1}{2}, 2 c-1 ; \frac{2 \sqrt{z}}{1+\sqrt{z}}\right)
$$

(see Beals-Wong [3, Section 8.9.6]). Putting $a=\frac{\beta}{2}, c=\frac{N}{2}$, we have

$$
\Phi_{\beta}(x, t)=(t+|x|)^{-\beta} F\left(\beta, \frac{N-1}{2}, N-1 ; \frac{2|x|}{t+|x|}\right)=t^{-\beta} F\left(\frac{\beta}{2}, \frac{\beta+1}{2}, \frac{N}{2} ; \frac{|x|^{2}}{t^{2}}\right) .
$$

This formula implies $\Phi_{\beta} \in C^{\infty}(Q)$. In one-dimensional case, the critical exponent for respective case does not appear. 
Then the family $\left\{\Phi_{\beta}\right\}_{\beta>0}$ satisfies the following properties. For detail, see [65] and also [22, 23].

Lemma 3.6. The following assertions hold:

(i) $\partial_{t}^{2} \Phi_{\beta}-\Delta \Phi_{\beta}=0$ on $Q$.

(ii) $\partial_{t} \Phi_{\beta}=-\beta \Phi_{\beta+1}$ on $Q$.

(iii) If $0 \leq \beta<\frac{N-1}{2}$, then $t^{-\beta} \leq \Phi_{\beta}(x, t) \leq K_{\beta} t^{-\beta}$ on $Q$.

(iv) If $\beta>\frac{N-1}{2}$, then

$$
k_{\beta} t^{-\beta}\left(1-\frac{|x|^{2}}{t^{2}}\right)^{\frac{N-1}{2}-\beta} \leq \Phi_{\beta}(x, t) \leq K_{\beta} t^{-\beta}\left(1-\frac{|x|^{2}}{t^{2}}\right)^{\frac{N-1}{2}-\beta}
$$

In view of the properties of $\left\{\Phi_{\beta}\right\}_{\beta>0}$, we will take $\Psi=\Phi_{\beta, \lambda} \psi_{R}$. Then we have the following lemma.

Lemma 3.7. Let $(f, g)$ satisfy (1.3) and let $u$ be a super-solution of $\partial_{t}^{2} u-\Delta u=H$ with $u(0)=\varepsilon f$, $\partial_{t} u(0)=\varepsilon g, H \in L^{2}\left(0, T ; L^{2}\left(\mathbb{R}^{N}\right)\right)$ and $\operatorname{supp} u \subset\left\{(x, t) \in \mathbb{R}^{N} \times[0, T] ;|x| \leq r_{0}+t\right\}$ for $r_{0}=\sup \{|x| ; x \in$ $\operatorname{supp}(f, g)\}$. Then for every $\beta>0$, there exists constants $\lambda_{\beta}>0, C_{\beta, 1}>0$ and $C_{\beta, 2}>0$ such that the followings hold:

(i) If $k \geq 2 p^{\prime}$ and $\beta>0$, then for every $\lambda \geq \lambda_{\beta}$ and $1 \leq R<T$

$$
\frac{1}{2} I[g] \varepsilon+\int_{0}^{T} \int_{\mathbb{R}^{N}} H \Phi_{\beta, \lambda} \psi_{R} d x d t \leq C_{\beta, 1} R^{-1} \int_{0}^{T} \int_{\mathbb{R}^{N}}|u| \Phi_{\beta+1, \lambda}\left[\psi_{R}^{*}\right]^{\frac{1}{p}} d x d t
$$

(ii) If $k \geq 2 p^{\prime}$ and $\beta>1$, then for every $\lambda \geq \lambda_{\beta}$ and $1 \leq R<T$

$$
\frac{1}{2} I[g] \varepsilon+\int_{0}^{T} \int_{\mathbb{R}^{N}} H \Phi_{\beta, \lambda} \psi_{R} d x d t \leq C_{\beta, 2} R^{-1} \int_{0}^{T} \int_{\mathbb{R}^{N}}\left|\partial_{t} u\right| \Phi_{\beta, \lambda}\left[\psi_{R}^{*}\right]^{\frac{1}{p}} d x d t
$$

Proof. Put

$$
c_{\beta, \lambda}(f, g)=\int_{\mathbb{R}^{N}} g \Phi_{\beta, \lambda}+\beta f \Phi_{\beta+1, \lambda} d x .
$$

Then we easily see that $c_{\beta, \lambda}(f, g) \rightarrow \int_{\mathbb{R}^{N}} g d x$ as $\lambda \rightarrow \infty$. Therefore there exists $\lambda_{\beta}>0$ such that for every $\lambda \geq \lambda_{\beta}$,

$$
c_{\beta, \lambda}(f, g) \geq \frac{1}{2} I[g] .
$$

Next, observe that for $R \geq 1$ and $(x, t) \in Q_{\lambda} \cap \operatorname{supp} \psi_{R}^{*}$,

$$
\begin{aligned}
\Phi_{\beta, \lambda}(x, t) & \leq 2 \lambda^{\beta}(\lambda+t)(\lambda+t+|x|)^{-\beta-1} F\left(\beta, \frac{N-1}{2}, N-1, \frac{2|x|}{\lambda+t+|x|}\right) \\
& \leq 2 \lambda^{\beta}(\lambda+t)(\lambda+t+|x|)^{-\beta-1} F\left(\beta+1, \frac{N-1}{2}, N-1, \frac{2|x|}{\lambda+t+|x|}\right) \\
& \leq 2 \lambda^{\beta+1}(1+t)(\lambda+t+|x|)^{-\beta-1} F\left(\beta+1, \frac{N-1}{2}, N-1, \frac{2|x|}{\lambda+t+|x|}\right) \\
& \leq 4 R \Phi_{\beta+1, \lambda}(x, t) .
\end{aligned}
$$


Therefore by Lemma 3.1 and Lemma 3.6 (i), (ii),

$$
\begin{aligned}
\left|\partial_{t}^{2}\left(\Phi_{\beta, \lambda} \psi_{R}\right)-\Delta\left(\Phi_{\beta, \lambda} \psi_{R}\right)\right| & \leq 2\left|\partial_{t} \Phi_{\beta, \lambda} \partial_{t} \psi_{R}\right|+\Phi_{\beta, \lambda}\left|\partial_{t}^{2} \psi_{R}\right| \\
& \leq C R^{-1} \Phi_{\beta, \lambda+1}\left[\psi_{R}^{*}\right]^{1-\frac{2}{k}}
\end{aligned}
$$

Choosing $\Psi=\Phi_{\beta, \lambda} \psi_{R}$ in Lemma 3.3, we have (i). For (ii), we choose $\Psi=-(\beta-1)^{-1} \lambda \Phi_{\beta-1, \lambda} \psi_{R}$ for $\beta>1$ and $\lambda>\lambda_{\beta}$ (the same choice as the case (i)). Noting that

$$
\partial_{t} \Psi=\Phi_{\beta, \lambda} \psi_{R}-\frac{\lambda}{\beta-1} \Phi_{\beta, \lambda} \partial_{t} \psi_{R} \geq \Phi_{\beta, \lambda} \psi_{R},
$$

we can deduce (ii).

Throughout the present paper we often use the following lemma.

Lemma 3.8. For every $R \geq 1$,

$$
\int_{\frac{R}{2}}^{R} \int_{B(0,1+t)} \Phi_{\beta, \lambda}^{p^{\prime}} d x d t \leq \begin{cases}C_{\lambda} R^{N+1-\beta p^{\prime}} & \text { if } \beta \in\left[0, \frac{N+1}{2}-\frac{1}{p}\right), \\ C_{\lambda} R^{N-\left(\frac{N-1}{2}\right) p^{\prime}} \log R & \text { if } \beta=\frac{N+1}{2}-\frac{1}{p}, \\ C_{\lambda} R^{N-\left(\frac{N-1}{2}\right) p^{\prime}} & \text { if } \beta \in\left(\frac{N+1}{2}-\frac{1}{p}, \infty\right) .\end{cases}
$$

Proof. All of the assertions are verified by using Lemma 3.6(iii) and (iv).

\subsection{Lemmas for lifespan estimates for respective critical cases}

To provide an upper bound of lifespan of solutions to respective critical case, we need to adopt the framework proposed in [24]. For the proof we refer the one of [24, Proposition 2.1].

Definition 3.3. For nonnegative function $w \in L_{\mathrm{loc}}^{1}\left([0, T) ; L^{1}\left(\mathbb{R}^{N}\right)\right)$, set

$$
Y[w](R)=\int_{0}^{R}\left(\int_{0}^{T} \int_{\mathbb{R}^{N}} w(x, t) \psi_{\sigma}^{*}(t) d x d t\right) \sigma^{-1} d \sigma, \quad R \in(0, T) .
$$

Then $Y[w]$ has the following properties.

Lemma 3.9. For $w \in L_{\mathrm{loc}}^{1}\left([0, T) ; L^{1}\left(\mathbb{R}^{N}\right)\right), Y[w](\cdot) \in C^{1}((0, T))$ and for every $R \in(0, T)$

$$
\begin{aligned}
\frac{d}{d R} Y[w](R) & =R^{-1} \int_{0}^{T} \int_{\mathbb{R}^{N}} w(x, t) \psi_{R}^{*}(t) d x d t, \\
Y[w](R) & \leq \int_{0}^{T} \int_{\mathbb{R}^{N}} w(x, t) \psi_{R}(t) d x d t .
\end{aligned}
$$

It worth noticing that in critical cases the behavior of $Y\left[|u|^{p} \Phi_{\beta}\right]$ is crucial to obtain not only the blowup phenomena but also the upper bound of lifespan for respective problems. The following lemma provides the sharp upper bound of solutions for respective problems.

Lemma 3.10. Let $2<t_{0}<T, 0 \leq \phi \in C^{1}\left(\left[t_{0}, T\right)\right)$. Assume that

$$
\begin{cases}\delta \leq K_{1} t \phi^{\prime}(t), & t \in\left(t_{0}, T\right), \\ \phi(t)^{p_{1}} \leq K_{2} t(\log t)^{p_{2}-1} \phi^{\prime}(t), & t \in\left(t_{0}, T\right)\end{cases}
$$


with $\delta, K_{1}, K_{2}>0$ and and $p_{1}, p_{2}>1$. If $p_{2}<p_{1}+1$, then there exists positive constants $\delta_{0}$ and $K_{3}$ (independent of $\delta$ ) such that

$$
T \leq \exp \left(K_{3} \delta^{-\frac{p_{1}-1}{p_{1}-p_{2}+1}}\right)
$$

when $0<\delta<\delta_{0}$.

Proof. If $T \leq t_{0}^{4}$, then we can choose $\delta_{0}^{\prime}=\left(4 K_{0}^{-1} \log t_{0}\right)^{-\frac{p_{1}-p_{2}+1}{p_{1}-1}}$. Therefore we assume $t_{0}^{4}<T$. By the first inequality in (3.13), we have for every $t \in\left(t_{0}^{2}, T\right)$,

$$
\phi(t)=\phi\left(t^{1 / 2}\right)+\int_{t^{1 / 2}}^{t} \phi^{\prime}(s) d s \geq \frac{\delta}{K_{1}}\left(\log t-\log t^{1 / 2}\right)=\frac{\delta}{2 K_{1}} \log t
$$

On the other hand, let $t_{1} \in\left(t_{0}^{4}, T\right)$ be arbitrary fixed. The second inequality in (3.13) implies

$$
\frac{d}{d t}[\phi(t)]^{1-p_{1}} \leq-\frac{p_{1}-1}{K_{2}} t^{-1}(\log t)^{1-p_{2}}, \quad t \in\left(t_{0}^{2}, T\right)
$$

and therefore integrating it over $\left[t_{1}^{1 / 2}, t_{1}\right]$, we deduce

$$
\begin{aligned}
{\left[\phi\left(t_{1}\right)\right]^{1-p_{1}} } & \leq\left[\phi\left(t_{1}^{1 / 2}\right)\right]^{1-p_{1}}-\frac{p_{1}-1}{K_{2}} \int_{t_{1}^{1 / 2}}^{t_{1}} s^{-1}(\log s)^{1-p_{2}} d s \\
& \leq\left[\frac{\delta}{4 K_{1}} \log t_{1}\right]^{1-p_{1}}-\frac{p_{1}-1}{K_{2}} \int_{1 / 2}^{1} \sigma^{1-p_{2}} d \sigma\left(\log t_{1}\right)^{2-p_{2}} \\
& \leq\left(\left[\frac{\delta}{4 K_{1}}\right]^{1-p_{1}}-\frac{p_{1}-1}{K_{2}} \int_{1 / 2}^{1} \sigma^{1-p_{2}} d \sigma\left(\log t_{1}\right)^{1+p_{1}-p_{2}}\right)\left(\log t_{1}\right)^{1-p_{1}} .
\end{aligned}
$$

This yields that

$$
\left(\log t_{1}\right)^{1+p_{1}-p_{2}} \leq \frac{\left(4 K_{1}\right)^{p_{1}-1} K_{2}}{p_{1}-1}\left(\int_{1 / 2}^{1} \sigma^{1-p_{2}} d \sigma\right)^{-1} \delta^{-\left(p_{1}-1\right)} .
$$

Since the choice of $t_{1} \in\left(t_{0}^{4}, T\right)$ is arbitrary, we obtain the desired upper bound of $T$.

\section{The case $\partial_{t}^{2} u-\Delta u=G(u)$}

The first problem is the following classical Cauchy problem of the following semilinear wave equation

$$
\begin{cases}\partial_{t}^{2} u-\Delta u=G(u), & (x, t) \in \mathbb{R}^{N} \times(0, T), \\ u(0)=\varepsilon f, & x \in \mathbb{R}^{N}, \\ \partial_{t} u(0)=\varepsilon g, & x \in \mathbb{R}^{N},\end{cases}
$$

where the nonlinearity $G \in C^{1}(\mathbb{R})$ satisfies

$$
G(0)=0, \quad G(s) \geq a|s|^{p}, \quad s \in \mathbb{R}
$$

for some $a>0$ and $p>1$. In this case the definition of weak solutions is the following: 
Definition 4.1. Let $f, g \in C_{c}^{\infty}\left(\mathbb{R}^{N}\right)$ and $p>1$. The function

$$
u \in C\left([0, T) ; H^{1}\left(\mathbb{R}^{N}\right)\right) \cap C^{1}\left([0, T) ; L^{2}\left(\mathbb{R}^{N}\right)\right), \quad G(u) \in L^{1}\left(0, T ; L^{1}\left(\mathbb{R}^{N}\right)\right)
$$

is called a weak solution of (4.1) in $(0, T)$ if $u(0)=\varepsilon f, \partial_{t} u(0)=\varepsilon g$ and for every $\Psi \in C_{c}^{\infty}\left(\mathbb{R}^{N} \times[0, T)\right.$,

$$
\begin{aligned}
& \varepsilon \int_{\mathbb{R}^{N}} g(x) \Psi(x, 0) d x+\int_{0}^{T} \int_{\mathbb{R}^{N}} G(u(x, t)) \Psi(x, t) d x d t \\
& =\int_{0}^{T} \int_{\mathbb{R}^{N}}\left(-\partial_{t} u(x, t) \partial_{t} \Psi(x, t)+\nabla u(x, t) \cdot \nabla \Psi(x, t)\right) d x d t .
\end{aligned}
$$

In order to give a unified view point with weakly coupled systems, we introduce

$$
\Gamma_{S}(N, p)=\left(1+\frac{1}{p}\right)(p-1)^{-1}-\frac{N-1}{2}=\frac{\gamma_{S}(N, p)}{2 p(p-1)} .
$$

The case of $G(s)=|s|^{p}$ with $1<p<p_{S}(N)$ was already shown in Section 2 . The essence of the proof for $1<p<p_{S}(N)$ is the same as in Section 2. Therefore we would state all assertions but prove only for the case $p=p_{S}(N)$. The assertion is formulated by the upper bound of maximal existence time of solutions to 4.1).

Proposition 4.1. Let $(f, g)$ satisfy (1.3) and let $u$ be a solution to (4.1) in $(0, T)$ satisfying supp $u \subset$ $\left\{(x, t) \in \mathbb{R}^{N} \times[0, T] ;|x| \leq r_{0}+t\right\}$ for $r_{0}=\sup \{|x| ; x \in \operatorname{supp}(f, g)\}$. If

$$
\Gamma_{S}(N, p) \geq 0
$$

(that is, $1<p \leq p_{S}(N)$ ), then $T$ has the following upper bound

$$
T \leq \begin{cases}C \varepsilon^{-\frac{p-1}{2}} & \text { if } N=1,1<p<\infty, \\ C \varepsilon^{-\frac{p-1}{3-p}} & \text { if } N=2,1<p \leq 2, \\ C \varepsilon^{-\Gamma_{S}(2, p)^{-1}} & \text { if } N=2,2<p<p_{S}(2), \\ C \varepsilon^{-\Gamma_{S}(N, p)^{-1}} & \text { if } N \geq 3,1<p<p_{S}(N), \\ \exp \left(C \varepsilon^{-p(p-1)}\right) & \text { if } N \geq 2, p=p_{S}(N)\end{cases}
$$

for every $\varepsilon \in\left(0, \varepsilon_{0}\right]$, where $\varepsilon_{0}$ and $C$ are positive constants independent of $\varepsilon$.

Proof. We only show the estimate for $T$ for the case $N \geq 2, p=p_{S}(N)$. We will deduce differential inequalities for $Y=Y\left[|u|^{p} \Phi_{\beta, \lambda}\right]$ defined in Lemma 3.9 with $\beta=\beta_{p}=\frac{N-1}{2}-\frac{1}{p}$ and $\lambda=\lambda_{\beta_{p}}$ (given in Lemma 3.77. By virtue of Lemma 3.9, we see from the inequality in Lemma 3.4 that

$$
\begin{aligned}
Y^{\prime}(R) R & =\int_{0}^{T} \int_{\mathbb{R}^{N}}|u|^{p} \Phi_{\beta, \lambda} \psi_{R}^{*} d x d t \\
& \geq\left(\frac{\lambda}{\lambda+1}\right)^{\beta} R^{-\beta} \int_{0}^{T} \int_{\mathbb{R}^{N}}|u|^{p} \psi_{R}^{*} d x d t \\
& \geq \delta_{1}\left(\frac{\lambda}{\lambda+1}\right)^{\beta}(\varepsilon I[g])^{p},
\end{aligned}
$$


where we used $\beta_{p}=N-\frac{N-1}{2} p$ by the assumption $\gamma_{S}(N, p)=0$. Moreover, using Lemma 3.7(i) with $\beta=\beta_{p}$ and Lemma 3.8 , we have

$$
\begin{aligned}
{[Y(R)]^{p} \leq\left(\int_{0}^{T} \int_{\mathbb{R}^{N}}|u|^{p} \Phi_{\beta, \lambda} \psi_{R} d x d t\right)^{p} } & \leq C R^{\frac{N-1}{2} p-N}(\log R)^{p-1} \int_{0}^{T} \int_{\mathbb{R}^{N}}|u|^{p} \psi_{R}^{*} d x d t \\
& \leq C(\log R)^{p-1} \int_{0}^{T} \int_{\mathbb{R}^{N}}|u|^{p} \Phi_{\beta, \lambda} \psi_{R}^{*} d x d t \\
& =C R(\log R)^{p-1} Y^{\prime}(R) .
\end{aligned}
$$

Applying Lemma 3.10 with $p_{1}=p_{2}=p$, we obtain $T \leq \exp \left(C \varepsilon^{-p(p-1)}\right)$. The proof is complete.

\section{The case $\partial_{t}^{2} u-\Delta u=G\left(\partial_{t} u\right)$}

In this section we consider the following semilinear wave equation with the nonlinearity governed by derivatives:

$$
\begin{cases}\partial_{t}^{2} u-\Delta u=G\left(\partial_{t} u\right) & (x, t) \in \mathbb{R}^{N} \times(0, T), \\ u(0)=\varepsilon f & x \in \mathbb{R}^{N}, \\ \partial_{t} u(0)=\varepsilon g & x \in \mathbb{R}^{N},\end{cases}
$$

where the nonlinearity $G \in C^{1}\left(\mathbb{R}^{N}\right)$ satisfies

$$
G(0)=0, \quad G(\sigma) \geq b|\sigma|^{p}, \quad \sigma \in \mathbb{R}
$$

for some $b>0$ and $p>1$. In this case the definition of weak solutions is the following:

Definition 5.1. Let $f, g \in C_{c}^{\infty}\left(\mathbb{R}^{N}\right)$ and $p>1$. The function

$$
u \in C\left([0, T) ; H^{1}\left(\mathbb{R}^{N}\right)\right) \cap C^{1}\left([0, T) ; L^{2}\left(\mathbb{R}^{N}\right)\right), \quad G\left(\partial_{t} u\right) \in L^{1}\left(0, T ; L^{1}\left(\mathbb{R}^{N}\right)\right)
$$

is called a weak solution of 5.1 in $(0, T)$ if $u(0)=\varepsilon f, \partial_{t} u(0)=\varepsilon g$ and for every $\Psi \in C_{c}^{\infty}\left(\mathbb{R}^{N} \times[0, T)\right.$,

$$
\begin{aligned}
& \varepsilon \int_{\mathbb{R}^{N}} g(x) \Psi(x, 0) d x+\int_{0}^{T} \int_{\mathbb{R}^{N}} G\left(\partial_{t} u(x, t)\right) \Psi(x, t) d x d t \\
& =\int_{0}^{T} \int_{\mathbb{R}^{N}}\left(-\partial_{t} u(x, t) \partial_{t} \Psi(x, t)+\nabla u(x, t) \cdot \nabla \Psi(x, t)\right) d x d t .
\end{aligned}
$$

For the problem (5.1), we set

$$
\Gamma_{G}(N, p)=\frac{1}{p-1}-\frac{n-1}{2} .
$$

The exponent $p_{G}(N)=\frac{N+1}{N-1}\left(\Gamma_{G}\left(N, p_{G}(N)\right)=0\right)$ is so-called Glassey exponent. For the convenience, we put $p_{G}(1)=\infty$.

Proposition 5.1. Let $(f, g)$ satisfy (1.3) and let $u$ be a solution to (5.1) in $(0, T)$ satisfying supp $u \subset$ $\left\{(x, t) \in \mathbb{R}^{N} \times[0, T] ;|x| \leq r_{0}+t\right\}$ for $r_{0}=\sup \{|x| ; x \in \operatorname{supp}(f, g)\}$. If

$$
\Gamma_{G}(N, p) \geq 0
$$


then $T$ has the following upper bound

$$
T \leq \begin{cases}C \varepsilon^{-\Gamma_{G}(N, p)^{-1}} & \text { if } 1<p<p_{G}(N), \\ \exp \left(C \varepsilon^{-(p-1)}\right) & \text { if } N \geq 2, p=p_{G}(N)\end{cases}
$$

for every $\varepsilon \in\left(0, \varepsilon_{0}\right]$, where $\varepsilon_{0}$ and $C$ are positive constants independent of $\varepsilon$.

Proof. Note that $u$ is a super-solution of $\partial_{t}^{2} u-\Delta u=b\left|\partial_{t} u\right|^{p}$. Choosing $\beta>\frac{N-1}{2}+1$ and $\lambda=\lambda_{\beta}$ in Lemma 3.7(ii) and Lemma 3.8, we have

$$
\begin{aligned}
& \frac{\varepsilon}{2} I[g]+b \int_{0}^{T} \int_{\mathbb{R}^{N}}\left|\partial_{t} u\right|^{p} \Phi_{\beta, \lambda} \psi_{R} d x d t \\
& \leq C R^{-1} \int_{0}^{T} \int_{\mathbb{R}^{N}}\left|\partial_{t} u\right| \Phi_{\beta, \lambda}\left[\psi_{R}^{*}\right]^{\frac{1}{p}} d x d t \\
& \leq C R^{-1}\left(\int_{0}^{T} \int_{\mathbb{R}^{N}}\left|\partial_{t} u\right|^{p} \Phi_{\beta, \lambda} \psi_{R}^{*} d x d t\right)^{\frac{1}{p}}\left(\int_{\frac{R}{2}}^{R} \int_{B\left(0, r_{0}+t\right)} \Phi_{\beta, \lambda} d x d t\right)^{\frac{1}{p^{\prime}}} \\
& \leq C R^{-\left(\frac{1}{p-1}-\frac{N-1}{2}\right) \frac{1}{p^{\prime}}}\left(\int_{0}^{T} \int_{\mathbb{R}^{N}}\left|\partial_{t} u\right|^{p} \Phi_{\beta, \lambda} \psi_{R}^{*} d x d t\right)^{\frac{1}{p}} .
\end{aligned}
$$

Setting $Y=Y\left[\left|\partial_{t} u\right|^{p} \Phi_{\beta, \lambda}\right]$ defined in Lemma3.9, we obtain

$$
\left(\frac{\varepsilon}{2} I[g]+b Y(R)\right)^{p} \leq C R^{-\Gamma_{G}(N, p)(p-1)+1} Y^{\prime}(R) .
$$

Solving the above differential inequality, we can deduce

$$
R \leq \begin{cases}C \varepsilon^{-\left(\frac{1}{p-1}-\frac{N-1}{2}\right)^{-1}} & \text { if } \Gamma_{G}(N, p)>0, \\ \exp \left(C \varepsilon^{-(p-1)}\right) & \text { if } \Gamma_{G}(N, p)=0 .\end{cases}
$$

Since the choice of $R \in(1, T)$ is arbitrary, we could derive the desired upper bound of $T$.

Remark 5.1. We can also see from $\psi_{R}^{*} \leq \psi_{R}$ that if $\Gamma_{G}(N, p)>0$, then by Young's inequality we have

$$
\frac{\varepsilon}{2} I[g] \leq C R^{-\left(\frac{1}{p-1}-\frac{N-1}{2}\right)}
$$

and therefore we can easily get the desired lifespan estimate for $1<p<\frac{N+1}{N-1}$. However, this argument does not work in the critical situation $p=\frac{N+1}{N-1}$.

\section{The case of a combined type $\partial_{t}^{2} u-\Delta u=G\left(u, \partial_{t} u\right)$}

In this section we discuss the semilinear equation with nonlinearity of a combined type

$$
\begin{cases}\partial_{t}^{2} u-\Delta u=G\left(u, \partial_{t} u\right) & (x, t) \in \mathbb{R}^{N} \times(0, T), \\ u(0)=\varepsilon f & x \in \mathbb{R}^{N}, \\ \partial_{t} u(0)=\varepsilon g & x \in \mathbb{R}^{N},\end{cases}
$$


where the nonlinearity $G \in C^{1}\left(\mathbb{R}^{2}\right)$ satisfies

$$
G(0,0)=0, \quad G(s, \sigma) \geq a|s|^{q}+b|\sigma|^{p} \quad(s, \sigma) \in \mathbb{R}^{2}
$$

for some $a, b>0$ and $p, q>1$. This problem has been considered by Zhou-Han [64] Hidano-WangYokoyama [19] and Wang-Zhou [56].

In this case the definition of weak solutions is the following:

Definition 6.1. Let $f, g \in C_{c}^{\infty}\left(\mathbb{R}^{N}\right)$ and $p>1$. The function

$$
u \in C\left([0, T) ; H^{1}\left(\mathbb{R}^{N}\right)\right) \cap C^{1}\left([0, T) ; L^{2}\left(\mathbb{R}^{N}\right)\right), \quad G\left(u, \partial_{t} u\right) \in L^{1}\left(0, T ; L^{1}\left(\mathbb{R}^{N}\right)\right)
$$

is called a weak solution of 6.1) in $(0, T)$ if $u(0)=\varepsilon f, \partial_{t} u(0)=\varepsilon g$ and for every $\Psi \in C_{c}^{\infty}\left(\mathbb{R}^{N} \times[0, T)\right)$,

$$
\begin{aligned}
& \varepsilon \int_{\mathbb{R}^{N}} g(x) \Psi(x, 0) d x+\int_{0}^{T} \int_{\mathbb{R}^{N}} G\left(u(x, t), \partial_{t} u(x, t)\right) \Psi(x, t) d x d t \\
& =\int_{0}^{T} \int_{\mathbb{R}^{N}}\left(-\partial_{t} u(x, t) \partial_{t} \Psi(x, t)+\nabla u(x, t) \cdot \nabla \Psi(x, t)\right) d x d t .
\end{aligned}
$$

For the problem 6.1 , we set

$$
\Gamma_{\mathrm{comb}}(N, p, q)=\frac{q+1}{p(q-1)}-\frac{N-1}{2} .
$$

The following assertion is already given by Hidano-Wang-Yokoyama [19].

Proposition 6.1. Let $(f, g)$ satisfy (1.3) and let $u$ be a solution to (6.1) in $(0, T)$ satisfying supp $u \subset$ $\left\{(x, t) \in \mathbb{R}^{N} \times[0, T) ;|x| \leq r_{0}+t\right\}$ for $r_{0}=\sup \{|x| ; x \in \operatorname{supp}(f, g)\}$. If

$$
\max \left\{\Gamma_{S}(N, q), \Gamma_{G}(N, p)\right\} \geq 0 \text { or } \Gamma_{\mathrm{comb}}(N, p, q)>0,
$$

then $T$ has the following upper bound

$$
T \leq \begin{cases}\exp \left(C \varepsilon^{-(p-1)}\right) & \text { if } p=\frac{N+1}{N-1}, q>1+\frac{4}{N-1}, \\ C \varepsilon^{-\Gamma_{G}(N, q)^{-1}} & \text { if } p<\frac{N+1}{N-1}, q>2 p-1, \\ C \varepsilon^{-\Gamma_{\mathrm{comb}}(N, p, q)^{-1}} & \text { if } p \leq q \leq 2 p-1, \Gamma_{\mathrm{comb}}(N, p, q)>0, \\ C \varepsilon^{-\Gamma_{S}(N, p)^{-1}} & \text { if } p>q, \quad q<p_{S}(N), \\ \exp \left(C \varepsilon^{q(q-1)}\right) & \text { if } p \geq q=p_{S}(N)\end{cases}
$$

for every $\varepsilon \in\left(0, \varepsilon_{0}\right]$, where $\varepsilon_{0}$ and $C$ are positive constants independent of $\varepsilon$.

Remark 6.1. In the case $\Gamma_{S}(N, q)<0, \Gamma_{G}(N, p)<0$ and $\Gamma_{\text {comb }}(N, p, q) \leq 0$, Hidano-Wang-Yokoyama [19] proved global existence of small solutions to 6.1) when $N=2,3$. Therefore although it is open but one can expect that the same conclusion can be proved for all dimensions.

Remark 6.2. In Wang-Zhou [56], the lower estimate for lifespan of solutions to [6.1) with $N=4$ and $p \in\{2\} \cup[3, \infty)$ is given. Therefore in these cases, the upper bound for $T$ in Proposition 6.1 is sharp. 
Proof. We have already proved the first, second, fourth and fifth cases in Propositions 4.1 and 5.1 because $G$ satisfies both $G \geq a|s|^{q}$ and $G \geq b|\sigma|^{q}$. Therefore we only consider the third case. Observe that $u$ is a super-solution of $\partial_{t}^{2} u-\Delta u=0$. By virtue of Lemma3.5, we already have

$$
\delta_{1}^{\prime}(I[g] \varepsilon)^{p} R^{N-\frac{N-1}{2} p} \leq \int_{0}^{T} \int_{\mathbb{R}^{N}}\left|\partial_{t} u\right|^{p} \psi_{R}^{*} d x d t .
$$

On the other hand, since $u$ is a super-solution of $\partial_{t}^{2} u-\Delta u \geq a|u|^{q}+b\left|\partial_{t} u\right|^{p}$, Lemma 3.2 with Young's inequality implies

$$
\begin{aligned}
I[g] \varepsilon+\int_{0}^{T} \int_{\mathbb{R}^{N}}\left(a|u|^{q}+b\left|\partial_{t} u\right|^{p}\right) \psi_{R}^{*} d x d t & \leq C R^{-2} \int_{0}^{T} \int_{\mathbb{R}^{N}}|u|\left[\psi_{R}^{*}\right]^{\frac{1}{q}} d x d t \\
& \leq \frac{a^{-\frac{1}{q-1}} C^{q^{\prime}}}{q^{\prime}} R^{N-\frac{q+1}{q-1}}+\frac{a}{q} \int_{0}^{T} \int_{\mathbb{R}^{N}}|u|^{q} \psi_{R}^{*} d x d t .
\end{aligned}
$$

Combining the above inequalities, we deduce

$$
b \delta_{1}^{\prime}(I[g] \varepsilon)^{p} R^{N-\frac{N-1}{2} p} \leq \frac{a^{-\frac{1}{q-1}} C^{q^{\prime}}}{q^{\prime}} R^{N-\frac{q+1}{q-1}} .
$$

Since the choice of $R \in(1, T)$ is arbitrary, this gives the third estimate for $T$.

\section{The case of the system $\partial_{t}^{2} u-\Delta u=G_{1}(v)$ and $\partial_{t}^{2} v-\Delta v=G_{2}(u)$}

The problem in this section is the following weakly coupled semilinear wave equations

$$
\begin{cases}\partial_{t}^{2} u-\Delta u=G_{1}(v), & (x, t) \in \mathbb{R}^{N} \times(0, T), \\ \partial_{t}^{2} v-\Delta v=G_{2}(u) & (x, t) \in \mathbb{R}^{N} \times(0, T), \\ u(0)=\varepsilon f_{1} & x \in \mathbb{R}^{N}, \\ \partial_{t} u(0)=\varepsilon g_{1} & x \in \mathbb{R}^{N}, \\ v(0)=\varepsilon f_{2} & x \in \mathbb{R}^{N}, \\ \partial_{t} v(0)=\varepsilon g_{2} & x \in \mathbb{R}^{N},\end{cases}
$$

where the nonlinearities $G_{1} \in C^{1}(\mathbb{R})$ and $G_{2} \in C^{1}(\mathbb{R})$ satisfy

$$
G_{1}(0)=0, \quad G_{2}(0)=0, \quad G_{1}(s) \geq a|s|^{p}, \quad G_{2}(s) \geq b|s|^{q} \quad s \in \mathbb{R}
$$

for some $a, b>0$ and $p, q>1$. The problem (7.1) with $G_{1}(s)=|s|^{p}$ and $G_{2}(s)=|s|^{q}$ is studied by Deng [7], Kubo-Ohta [31], Agemi-Kurokawa-Takamura [2], Kurokawa-Takamura-Wakasa [35]. The aim of this section is to find the same result about upper bound of lifespan of solutions to (7.1) by using a test function method similar to the one in Section 4

In this case the definition of weak solutions is the following:

Definition 7.1. Let $f_{1}, f_{2}, g_{1}, g_{2} \in C_{c}^{\infty}\left(\mathbb{R}^{N}\right)$. The pair of functions

$$
\begin{aligned}
& (u, v) \in C\left([0, T) ;\left(H^{1}\left(\mathbb{R}^{N}\right)\right)^{2}\right) \cap C^{1}\left([0, T) ;\left(L^{2}\left(\mathbb{R}^{N}\right)\right)^{2}\right), \\
& G_{2}(u) \in L^{1}\left(0, T ; L^{1}\left(\mathbb{R}^{N}\right)\right), \quad G_{1}(v) \in L^{1}\left(0, T ; L^{1}\left(\mathbb{R}^{N}\right)\right)
\end{aligned}
$$


is called a weak solution of (7.1) in $(0, T)$ if $(u, v)(0)=\left(\varepsilon f_{1}, \varepsilon f_{2}\right),\left(\partial_{t} u, \partial_{t} v\right)(0)=\left(\varepsilon g_{1}, \varepsilon g_{2}\right)$ and for every $\Psi \in C_{c}^{\infty}\left(\mathbb{R}^{N} \times[0, T)\right)$

$$
\begin{aligned}
& \varepsilon \int_{\mathbb{R}^{N}} g_{1}(x) \Psi(x, 0) d x+\int_{0}^{T} \int_{\mathbb{R}^{N}} G_{1}(v(x, t)) \Psi(x, t) d x d t \\
& =\int_{0}^{T} \int_{\mathbb{R}^{N}}\left(-\partial_{t} u(x, t) \partial_{t} \Psi(x, t)+\nabla u(x, t) \cdot \nabla \Psi(x, t)\right) d x d t, \\
& \varepsilon \int_{\mathbb{R}^{N}} g_{2}(x) \Psi(x, 0) d x+\int_{0}^{T} \int_{\mathbb{R}^{N}} G_{2}(u(x, t)) \Psi(x, t) d x d t \\
& =\int_{0}^{T} \int_{\mathbb{R}^{N}}\left(-\partial_{t} u(x, t) \partial_{t} \Psi(x, t)+\nabla u(x, t) \cdot \nabla \Psi(x, t)\right) d x d t .
\end{aligned}
$$

As in the previous works listed above, we introduce

$$
F_{S S}(N, p, q)=\left(p+2+\frac{1}{q}\right)(p q-1)^{-1}-\frac{N-1}{2} .
$$

The assertion for the estimates for $T$ is the following. The result has been given until KurokawaTakamura-Wakasa [35].

Proposition 7.1. Let $\left(f_{1}, g_{1}\right)$ and $\left(f_{2}, g_{2}\right)$ satisfy (1.3) and let $(u, v)$ be a weak solution of the system (7.1) satisfying $\operatorname{supp}(u, v) \subset\left\{(x, t) \in \mathbb{R}^{N} \times[0, T) ;|x| \leq r_{0}+t\right\}$ for $r_{0}=\sup \left\{|x| ; x \in \operatorname{supp}\left(f_{1}, f_{2}, g_{1}, g_{2}\right)\right\}$. If

$$
\Gamma_{S S}(N, p, q)=\max \left\{F_{S S}(N, p, q), F_{S S}(N, q, p)\right\} \geq 0,
$$

then $T$ has the following upper bound

$$
T \leq \begin{cases}C \varepsilon^{-\Gamma_{S S}(N, p, q)^{-1}} & \text { if } \Gamma_{S S}(N, p, q)>0, \\ \exp \left(C \varepsilon^{-\min \{p(p q-1), q(p q-1)\}}\right) & \text { if } \Gamma_{S S}(N, p, q)=0, p \neq q, \\ \exp \left(C \varepsilon^{-p(p-1)}\right) & \text { if } \Gamma_{S S}(N, p, q)=0, p=q\end{cases}
$$

for every $\varepsilon \in\left(0, \varepsilon_{0}\right]$, where $\varepsilon_{0}$ and $C$ are positive constants independent of $\varepsilon$.

Proof. We assume $F_{S S}(N, p, q) \geq F_{S S}(N, q, p)$, otherwise, we can interchange $u$ and $v$. Moreover, we already have the following estimates by Lemma 3.4 .

$$
\delta_{1}\left(I\left[g_{1}\right] \varepsilon\right)^{q} R^{N-\frac{N-1}{2} q} \leq \int_{0}^{T} \int_{\mathbb{R}^{N}}|u|^{q} \psi_{R}^{*} d x d t .
$$

Now we consider the case $F_{S S}(N, p, q)>0$. By Lemma 3.2. we have

$$
\begin{aligned}
& \left(\int_{0}^{T} \int_{\mathbb{R}^{N}}|v|^{p} \psi_{R} d x d t\right)^{q} \leq C R^{-2+(N-1)(q-1)} \int_{0}^{T} \int_{\mathbb{R}^{N}}|u|^{q} \psi_{R}^{*} d x d t, \\
& \left(\int_{0}^{T} \int_{\mathbb{R}^{N}}|u|^{q} \psi_{R} d x d t\right)^{p} \leq C R^{-2+(N-1)(p-1)} \int_{0}^{T} \int_{\mathbb{R}^{N}}|\nu|^{p} \psi_{R}^{*} d x d t .
\end{aligned}
$$


These imply

$$
\begin{aligned}
\left(\int_{0}^{T} \int_{\mathbb{R}^{N}}|u|^{q} \psi_{R} d x d t\right)^{p q} & \leq C\left(R^{-2+(N-1)(p-1)} \int_{\mathbb{R}^{N}}|v|^{p} \psi_{R}^{*} d x d t\right)^{q} \\
& \leq C R^{[-2+(N-1)(p-1)] q-2+(N-1)(q-1)} \int_{0}^{T} \int_{\mathbb{R}^{N}}|u|^{q} \psi_{R}^{*} d x d t \\
& \leq C R^{(N-1)(p q-1)-2(q+1)} \int_{0}^{T} \int_{\mathbb{R}^{N}}|u|^{q} \psi_{R}^{*} d x d t,
\end{aligned}
$$

and hence

$$
\int_{0}^{T} \int_{\mathbb{R}^{N}}|u|^{q} \psi_{R} d x d t \leq C R^{N-1-\frac{2(q+1)}{p q-1}}=C R^{N-\frac{p q+2 q+1}{p q-1}} .
$$

Combining (7.2), we deduce

$$
\left(I\left[g_{1}\right] \varepsilon\right)^{q} \leq C R^{\frac{N-1}{2} q-\frac{p q+2 q+1}{p q-1}}=C R^{-q F_{S S}(N, p, q)} .
$$

Since $R \in(1, T)$ is arbitrary, we have the upper bound for $T$.

Next we consider the critical case $F_{S S}(N, p, q)=0$. If $F_{S S}(N, p, q)=F_{S S}(N, q, p)$, then we have $p=q=p_{S}(N)$. In this case, we consider the following differential inequality

$$
\partial_{t}^{2}(u+v)-\Delta(u+v)=b|u|^{p}+a|v|^{p} \geq 2^{-p} \min \{a, b\}(|u|+|v|)^{p} \geq 2^{-p} \min \{a, b\}|u+v|^{p} .
$$

Applying Proposition 4.1 with $a$ replaced with $2^{-p} \min \{a, b\}$, we can obtain $T \leq \exp \left(C \varepsilon^{-p(p-1)}\right)$. Here we assume $0=F_{S S}(N, p, q)>F_{S S}(N, q, p)$. Then combining (7.2) and (7.3), we have

$$
\int_{0}^{T} \int_{\mathbb{R}^{N}}|\nu|^{p} \psi_{R}^{*} d x d t \geq \delta_{1}^{\prime}\left(I\left[g_{1}\right] \varepsilon\right)^{p q} R^{\left(N-\frac{N-1}{2} q\right) p+2-(N-1)(p-1)}=\delta_{1}^{\prime}\left(I\left[g_{1}\right] \varepsilon\right)^{p q} R^{\frac{N-1}{2}-\frac{1}{q}}
$$

where we have used $F_{S S}(N, p, q)=0$. We see from Lemma3.6(iii) that

$$
\int_{0}^{T} \int_{\mathbb{R}^{N}}|v|^{p} \Phi_{\beta, \lambda} \psi_{R}^{*} d x d t \geq \delta_{1}^{\prime}\left(I\left[g_{1}\right] \varepsilon\right)^{p q}
$$

with $\beta=\beta_{q}=\frac{N-1}{2}-\frac{1}{q}$ and $\lambda=\lambda_{\beta_{q}}$. By using Lemma 3.7 (i) with $\beta=\beta_{q}$, Lemma 3.8, (7.3) and the condition $F_{S S}(N, p, q)=0$, we have

$$
\begin{aligned}
\left(\int_{0}^{T} \int_{\mathbb{R}^{N}}|v|^{p} \Phi_{\beta, \lambda} \psi_{R} d x d t\right)^{p q} & \leq C R^{-\left(N-\frac{N-1}{2} q\right) p}(\log R)^{p(q-1)}\left(\int_{0}^{T} \int_{\mathbb{R}^{N}}|u|^{q} \psi_{R}^{*} d x d t\right)^{p} \\
& \leq C R^{-\left(N-\frac{N-1}{2} q\right) p-2+(N-1)(p-1)}(\log R)^{p(q-1)} \int_{0}^{T} \int_{\mathbb{R}^{N}}|v|^{p} \psi_{R}^{*} d x d t \\
& \leq C(\log R)^{p(q-1)} \int_{0}^{T} \int_{\mathbb{R}^{N}}|v|^{p} \Phi_{\beta, \lambda} \psi_{R}^{*} d x d t .
\end{aligned}
$$

In view of Lemma 3.9, taking $Y=Y\left[|v|^{p} \Phi_{\beta, \lambda}\right]$, we deduce

$$
\left\{\begin{array}{l}
\varepsilon^{p q} \leq C R Y^{\prime}(R), \\
{[Y(R)]^{p q} \leq C R(\log R)^{p(q-1)} Y^{\prime}(R) .}
\end{array}\right.
$$

Applying Lemma 3.10 with $\delta=\varepsilon^{p q}, p_{1}=p q$ and $p_{2}=p(q-1)+1$, we obtain

$$
T \leq \exp \left(C \varepsilon^{-q(p q-1)}\right) .
$$

The proof is complete. 


\section{The case of the system $\partial_{t}^{2} u-\Delta u=G_{1}\left(\partial_{t} v\right)$ and $\partial_{t}^{2} v-\Delta v=G_{2}\left(\partial_{t} u\right)$}

We consider the following weakly coupled system of semilinear wave equations with nonlinearities including derivatives

$$
\begin{cases}\partial_{t}^{2} u-\Delta u=G_{1}\left(\partial_{t} v\right), & (x, t) \in \mathbb{R}^{N} \times(0, T), \\ \partial_{t}^{2} v-\Delta v=G_{2}\left(\partial_{t} u\right) & (x, t) \in \mathbb{R}^{N} \times(0, T), \\ u(0)=\varepsilon f_{1} & x \in \mathbb{R}^{N}, \\ \partial_{t} u(0)=\varepsilon g_{1} & x \in \mathbb{R}^{N}, \\ v(0)=\varepsilon f_{2} & x \in \mathbb{R}^{N}, \\ \partial_{t} v(0)=\varepsilon g_{2} & x \in \mathbb{R}^{N},\end{cases}
$$

where the nonlinearities $G_{1} \in C^{1}(\mathbb{R})$ and $G_{2} \in C^{1}(\mathbb{R})$ satisfy

$$
G_{1}(0)=0, \quad G_{2}(0)=0, \quad G_{1}(\sigma) \geq a|\sigma|^{p}, \quad G_{2}(\sigma) \geq b|\sigma|^{q}, \quad \sigma \in \mathbb{R}
$$

for some $a, b>0$ and $p, q>1$. The blowup phenomena of the system (8.1) is studied in Deng [7]. It seems that the upper bound of lifespan of solutions to 18.1 has not been obtained so far. In the present paper we obtain an upper bound of lifespan by our technique similar to Section 5 .

In this case the definition of weak solutions is the following:

Definition 8.1. Let $f_{1}, f_{2}, g_{1}, g_{2} \in C_{c}^{\infty}\left(\mathbb{R}^{N}\right)$. The pair of functions

$$
\begin{gathered}
(u, v) \in C\left([0, T) ;\left(H^{1}\left(\mathbb{R}^{N}\right)\right)^{2}\right) \cap C^{1}\left([0, T) ;\left(L^{2}\left(\mathbb{R}^{N}\right)\right)^{2}\right), \\
G_{2}\left(\partial_{t} u\right) \in L^{1}\left(0, T ; L^{1}\left(\mathbb{R}^{N}\right)\right), \quad G_{1}\left(\partial_{t} v\right) \in L^{1}\left(0, T ; L^{1}\left(\mathbb{R}^{N}\right)\right)
\end{gathered}
$$

is called a weak solution of 8.1 in $(0, T)$ if $(u, v)(0)=\left(\varepsilon f_{1}, \varepsilon f_{2}\right),\left(\partial_{t} u, \partial_{t} v\right)(0)=\left(\varepsilon g_{1}, \varepsilon g_{2}\right)$ and for every $\Psi \in C_{c}^{\infty}\left(\mathbb{R}^{N} \times[0, T)\right)$

$$
\begin{aligned}
& \varepsilon \int_{\mathbb{R}^{N}} g_{1}(x) \Psi(x, 0) d x+\int_{0}^{T} \int_{\mathbb{R}^{N}} G_{1}\left(\partial_{t} v(x, t)\right) \Psi(x, t) d x d t \\
& =\int_{0}^{T} \int_{\mathbb{R}^{N}}\left(-\partial_{t} u(x, t) \partial_{t} \Psi(x, t)+\nabla u(x, t) \cdot \nabla \Psi(x, t)\right) d x d t \\
& \varepsilon \int_{\mathbb{R}^{N}} g_{2}(x) \Psi(x, 0) d x+\int_{0}^{T} \int_{\mathbb{R}^{N}} G_{2}\left(\partial_{t} u(x, t)\right) \Psi(x, t) d x d t \\
& =\int_{0}^{T} \int_{\mathbb{R}^{N}}\left(-\partial_{t} u(x, t) \partial_{t} \Psi(x, t)+\nabla u(x, t) \cdot \nabla \Psi(x, t)\right) d x d t .
\end{aligned}
$$

In this case, set

$$
F_{G G}(N, p, q)=\frac{p+1}{p q-1}-\frac{N-1}{2} .
$$

The exponent $F_{G G}$ seems to play the same rule (with the shift of dimension $N$ to $N-1$ ) as the one for weakly coupled heat equations in Escobedo-Herrero [9] (see also Nishihara-Wakasugi [44] for weakly coupled damped wave equations).

Proposition 8.1. Let $\left(f_{1}, g_{1}\right)$ and $\left(f_{2}, g_{2}\right)$ satisfy (1.3) and let $(u, v)$ be a weak solution of the system (8.1) satisfying $\operatorname{supp}(u, v) \subset\left\{(x, t) \in \mathbb{R}^{N} \times[0, T) ;|x| \leq r_{0}+t\right\}$ for $r_{0}=\sup \left\{|x| ; x \in \operatorname{supp}\left(f_{1}, f_{2}, g_{1}, g_{2}\right)\right\}$. If

$$
\Gamma_{G G}(N, p, q)=\max \left\{F_{G G}(N, p, q), F_{G G}(N, q, p)\right\} \geq 0,
$$


then $T$ has the following upper bound

$$
T \leq\left\{\begin{array}{lll}
C \varepsilon^{-\Gamma_{G G}(N, p, q)^{-1}} & \text { if } \Gamma_{G G}(N, p, q)>0, & \\
\exp \left(C \varepsilon^{-(p q-1)}\right) & \text { if } \Gamma_{G G}(N, p, q)=0, & p \neq q, \\
\exp \left(C \varepsilon^{-(p-1)}\right) & \text { if } \Gamma_{G G}(N, p, q)=0, & p=q
\end{array}\right.
$$

for every $\varepsilon \in\left(0, \varepsilon_{0}\right]$, where $\varepsilon_{0}$ and $C$ are positive constants independent of $\varepsilon$.

Proof. As in the proof of Proposition 7.1, we only consider the case $F_{G G}(N, p, q) \geq F_{G G}(N, q, p)$ (that is, $p \geq q$ ). Lemma 3.7 (ii) with $\beta>\frac{N-1}{2}+1$ and $\lambda=\lambda_{\beta}$ and Lemma 3.8 imply

$$
\begin{aligned}
\frac{\varepsilon}{2} I\left[g_{1}\right]+a \int_{0}^{T} \int_{\mathbb{R}^{N}}\left|\partial_{t} \nu\right|^{p} \Phi_{\beta, \lambda} \psi_{R} d x d t & \leq C R^{-1} \int_{0}^{T} \int_{\mathbb{R}^{N}}\left|\partial_{t} u\right| \Phi_{\beta, \lambda}\left[\psi_{R}^{*}\right]^{\frac{1}{q}} d x d t \\
& \leq C R^{-\left(\frac{1}{q-1}-\frac{N-1}{2}\right) \frac{1}{q^{\prime}}}\left(\int_{0}^{T} \int_{\mathbb{R}^{N}}\left|\partial_{t} u\right|^{q} \Phi_{\beta, \lambda} \psi_{R}^{*} d x d t\right)^{\frac{1}{q}},
\end{aligned}
$$

and similarly,

$$
\begin{aligned}
\frac{\varepsilon}{2} I\left[g_{2}\right]+b \int_{0}^{T} \int_{\mathbb{R}^{N}}\left|\partial_{t} u\right|^{q} \Phi_{\beta, \lambda} \psi_{R} d x d t & \leq C R^{-1} \int_{0}^{T} \int_{\mathbb{R}^{N}}\left|\partial_{t} v\right| \Phi_{\beta, \lambda}\left[\psi_{R}^{*}\right]^{\frac{1}{p}} d x d t \\
& \leq C R^{-\left(\frac{1}{p-1}-\frac{N-1}{2}\right) \frac{1}{p^{\prime}}}\left(\int_{0}^{T} \int_{\mathbb{R}^{N}}\left|\partial_{t} v\right|^{p} \Phi_{\beta, \lambda} \psi_{R}^{*} d x d t\right)^{\frac{1}{p}} .
\end{aligned}
$$

Combining these inequalities, we deduce

$$
\left(\frac{\varepsilon}{2} I\left[g_{1}\right]+a \int_{0}^{T} \int_{\mathbb{R}^{N}}\left|\partial_{t} \nu\right|^{p} \Phi_{\beta, \lambda} \psi_{R} d x d t\right)^{p q} \leq C R^{\left(\frac{N-1}{2}\right)(p q-1)-p-1} \int_{0}^{T} \int_{\mathbb{R}^{N}}\left|\partial_{t} \nu\right|^{p} \Phi_{\beta, \lambda} \psi_{R}^{*} d x d t .
$$

Using $Y=Y\left[\left|\partial_{t} v\right|^{p} \Phi_{\beta, \lambda}\right]$ in Lemma 3.9 , we can verify

$$
T \leq \begin{cases}C \varepsilon^{-F_{G G}(N, p, q)^{-1}} & \text { if } F_{G G}(N, p, q)>0, \\ \exp \left(C \varepsilon^{-(p q-1)}\right) & \text { if } F_{G G}(N, p, q)=0 .\end{cases}
$$

Note that in the case $F_{G G}(N, p, q)=F_{G G}(N, q, p)=0$, we have $p=q$ and then $\Gamma_{G}(N, p)=0$. Applying Proposition 5.1 to the inequality

$$
\partial_{t}^{2}(u+v)-\Delta(u+v) \geq 2^{-p} \min \{a, b\}\left|\partial_{t}(u+v)\right|^{p},
$$

we have $T \leq \exp \left(C \varepsilon^{-(p-1)}\right)$.

\section{The case of system $\partial_{t}^{2} u-\Delta u=G_{1}(v)$ and $\partial_{t}^{2} v-\Delta v=G_{2}\left(\partial_{t} u\right)$}

To close the paper, in the last section we consider the weakly coupled system of semilinear wave equations of the form

$$
\begin{cases}\partial_{t}^{2} u-\Delta u=G_{1}(v), & (x, t) \in \mathbb{R}^{N} \times(0, T), \\ \partial_{t}^{2} v-\Delta v=G_{2}\left(\partial_{t} u\right) & (x, t) \in \mathbb{R}^{N} \times(0, T), \\ u(0)=\varepsilon f_{1} & x \in \mathbb{R}^{N}, \\ \partial_{t} u(0)=\varepsilon g_{1} & x \in \mathbb{R}^{N}, \\ v(0)=\varepsilon f_{2} & x \in \mathbb{R}^{N}, \\ \partial_{t} v(0)=\varepsilon g_{2} & x \in \mathbb{R}^{N},\end{cases}
$$


where the nonlinearities $G_{1} \in C^{1}(\mathbb{R})$ and $G_{2} \in C^{1}(\mathbb{R})$ satisfy

$$
G_{1}(0)=0, \quad G_{2}(0)=0, \quad G_{1}(s) \geq a|s|^{q}, \quad G_{2}(\sigma) \geq b|\sigma|^{p}, \quad s, \sigma \in \mathbb{R}
$$

for some $a, b>0$ and $p, q>1$. In this case the definition of weak solutions is the following:

Definition 9.1. Let $f_{1}, f_{2}, g_{1}, g_{2} \in C_{c}^{\infty}\left(\mathbb{R}^{N}\right)$. The pair of functions $(u, v)$

$$
\begin{gathered}
(u, v) \in C\left([0, T) ;\left(H^{1}\left(\mathbb{R}^{N}\right)\right)^{2}\right) \cap C^{1}\left([0, T) ;\left(L^{2}\left(\mathbb{R}^{N}\right)\right)^{2}\right), \\
G_{2}\left(\partial_{t} u\right) \in L^{1}\left(0, T ; L^{1}\left(\mathbb{R}^{N}\right)\right), \quad G_{1}(v) \in L^{1}\left(0, T ; L^{1}\left(\mathbb{R}^{N}\right)\right)
\end{gathered}
$$

is called a weak solution of 9.1$)$ in $(0, T)$ if $(u, v)(0)=\left(\varepsilon f_{1}, \varepsilon f_{2}\right),\left(\partial_{t} u, \partial_{t} v\right)(0)=\left(\varepsilon g_{1}, \varepsilon g_{2}\right)$ and for every $\Psi \in C_{c}^{\infty}\left(\mathbb{R}^{N} \times[0, T)\right)$

$$
\begin{aligned}
& \varepsilon \int_{\mathbb{R}^{N}} g_{1}(x) \Psi(x, 0) d x+\int_{0}^{T} \int_{\mathbb{R}^{N}} G_{1}(v(x, t)) \Psi(x, t) d x d t \\
& =\int_{0}^{T} \int_{\mathbb{R}^{N}}\left(-\partial_{t} u(x, t) \partial_{t} \Psi(x, t)+\nabla u(x, t) \cdot \nabla \Psi(x, t)\right) d x d t, \\
& \varepsilon \int_{\mathbb{R}^{N}} g_{2}(x) \Psi(x, 0) d x+\int_{0}^{T} \int_{\mathbb{R}^{N}} G_{2}\left(\partial_{t} u(x, t)\right) \Psi(x, t) d x d t \\
& =\int_{0}^{T} \int_{\mathbb{R}^{N}}\left(-\partial_{t} u(x, t) \partial_{t} \Psi(x, t)+\nabla u(x, t) \cdot \nabla \Psi(x, t)\right) d x d t .
\end{aligned}
$$

Here we introduce two kind of exponent for the problem (9.1).

$$
\begin{aligned}
& F_{S G, 1}(N, p, q)=\left(\frac{1}{p}+1+q\right)(p q-1)^{-1}-\frac{N-1}{2}, \\
& F_{S G, 2}(N, p, q)=\left(2+\frac{1}{q}\right)(p q-1)^{-1}-\frac{N-1}{2} .
\end{aligned}
$$

The problem (9.1) is recently discussed in Hidano-Yokoyama [20] and the blowup phenomena for small solutions are shown in the case $F_{S G, 1}(N, p, q)>0$. The other condition $F_{S G, 2}(N, p, q) \geq 0$ is now carried out by our test function method. Furthermore, we can also find the lifespan estimate for $(p, q)$ on the borderline case.

Proposition 9.1. Let $\left(f_{1}, g_{1}\right)$ and $\left(f_{2}, g_{2}\right)$ satisfy (1.3) and let $(u, v)$ be a weak solution of the system (9.1) satisfying $\operatorname{supp}(u, v) \subset\left\{(x, t) \in \mathbb{R}^{N} \times[0, T) ;|x| \leq r_{0}+t\right\}$ for $r_{0}=\sup \left\{|x| ; x \in \operatorname{supp}\left(f_{1}, f_{2}, g_{1}, g_{2}\right)\right\}$. If

$$
\Gamma_{S G}(N, p, q)=\max \left\{F_{S G, 1}(N, p, q), F_{S G, 2}(N, p, q)\right\} \geq 0,
$$

then $T$ has the following upper bound:

$$
T \leq \begin{cases}C \varepsilon^{-\Gamma_{S G}(N, p, q)^{-1}} & \text { if } \Gamma_{S G}(N, p, q)>0, \\ \exp \left(C \varepsilon^{-q(p q-1)}\right) & \text { if } \Gamma_{S G, 1}(N, p, q)=0>F_{S G, 2}(N, p, q), \\ \exp \left(C \varepsilon^{-p(p q-1)}\right) & \text { if } \Gamma_{S G, 1}(N, p, q)<0=F_{S G, 2}(N, p, q), \\ \exp \left(C \varepsilon^{-(p q-1)}\right) & \text { if } \Gamma_{S G, 1}(N, p, q)=0=F_{S G, 2}(N, p, q)\end{cases}
$$

for every $\varepsilon \in\left(0, \varepsilon_{0}\right]$, where $\varepsilon_{0}$ and $C$ are positive constants independent of $\varepsilon$. 
Remark 9.1. On the critical curve, we could find lifespan estimates including exponential functions. At the intersection point of two critical curves $\left\{\Gamma_{S G, 1}=0\right\}$ and $\left\{\Gamma_{S G, 2}=0\right\}$, some discontinuity in the sense of lifespan estimates appears.

Proof. (The case $\Gamma_{S G}(N, p, q)>0$ ). By Lemma3.4 for $v$ and Lemma 3.5 for $\partial_{t} u$, we have

$$
\begin{aligned}
& \delta_{1}\left(I\left[g_{1}\right] \varepsilon\right)^{p} R^{N-\frac{N-1}{2} p} \leq \int_{0}^{T} \int_{\mathbb{R}^{N}}\left|\partial_{t} u\right|^{p} \psi_{R}^{*} d x d t, \\
& \delta_{1}^{\prime}\left(I\left[g_{2}\right] \varepsilon\right)^{q} R^{N-\frac{N-1}{2} q} \leq \int_{0}^{T} \int_{\mathbb{R}^{N}}|v|^{q} \psi_{R}^{*} d x d t .
\end{aligned}
$$

On the other hand, since $u$ is a super-solution of $\partial_{t}^{2} u-\Delta u=G=|v|^{q}$ and $v$ is a super-solution of $\partial_{t}^{2} v-\Delta v=\widetilde{G}=\left|\partial_{t} u\right|^{p}$ using Lemma 3.2 (ii) with $u$, we have

$$
\begin{aligned}
\int_{0}^{T} \int_{\mathbb{R}^{N}}|v|^{q} \psi_{R} d x d t & \leq C R^{-1} \int_{0}^{T} \int_{\mathbb{R}^{N}}\left|\partial_{t} u\right|\left[\psi_{R}^{*}\right]^{\frac{1}{p}} d x d t \\
& \leq C R^{\frac{-1+N(p-1)}{p}}\left(\int_{0}^{T} \int_{\mathbb{R}^{N}}\left|\partial_{t} u\right|^{p} \psi_{R}^{*} d x d t\right)^{\frac{1}{p}}
\end{aligned}
$$

and using Lemma 3.2 (i) for $v$, we have

$$
\begin{aligned}
\int_{0}^{T} \int_{\mathbb{R}^{N}}\left|\partial_{t} u\right|^{p} \psi_{R} d x d t & \leq C R^{-2} \int_{0}^{T} \int_{\mathbb{R}^{N}}|v|\left[\psi_{R}^{*}\right]^{\frac{1}{q}} d x d t \\
& \leq C R^{\frac{-2+(N-1)(q-1)}{q}}\left(\int_{0}^{T} \int_{\mathbb{R}^{N}}|v|^{q} \psi_{R}^{*} d x d t\right)^{\frac{1}{q}} .
\end{aligned}
$$

Combining the above inequalities, we deduce

$$
\begin{aligned}
\left(\int_{0}^{T} \int_{\mathbb{R}^{N}}\left|\partial_{t} u\right|^{p} \psi_{R} d x d t\right)^{p q} & \leq C R^{[-2+(N-1)(q-1)] p-1+N(p-1)} \int_{0}^{T} \int_{\mathbb{R}^{N}}\left|\partial_{t} u\right|^{p} \psi_{R}^{*} d x d t \\
& \leq C R^{-p q-p-1+N(p q-1)} \int_{0}^{T} \int_{\mathbb{R}^{N}}\left|\partial_{t} u\right|^{p} \psi_{R}^{*} d x d t
\end{aligned}
$$

and

$$
\begin{aligned}
\left(\int_{0}^{T} \int_{\mathbb{R}^{N}}|\nu|^{q} \psi_{R} d x d t\right)^{p q} & \leq C R^{[-1+N(p-1)] q-2+(N-1)(q-1)} \int_{0}^{T} \int_{\mathbb{R}^{N}}|\nu|^{q} \psi_{R}^{*} d x d t \\
& \leq C R^{-2 q-1+N(p q-1)} \int_{0}^{T} \int_{\mathbb{R}^{N}}|\nu|^{q} \psi_{R}^{*} d x d t .
\end{aligned}
$$

These yield that

$$
\begin{gathered}
\int_{0}^{T} \int_{\mathbb{R}^{N}}\left|\partial_{t} u\right|^{p} \psi_{R} d x d t \leq C R^{N-\frac{p q+p+1}{p q-1}} \\
\int_{0}^{T} \int_{\mathbb{R}^{N}}|v|^{q} \psi_{R} d x d t \leq C R^{N-\frac{2 q+1}{p q-1}}
\end{gathered}
$$

and therefore combining (9.2) and (9.3), we obtain the desired estimates for $T$ for $\Gamma_{S G}(N, p, q)>0$. 
(The case $\left.F_{S G, 1}(N, p, q)=0>F_{S G, 2}(N, p, q)\right)$. Observe that the condition $F_{S G, 1}(N, p, q)=0$ yields

$$
\left(N-\frac{N-1}{2} p-\beta_{q}\right) q+\left(N-\frac{N-1}{2} q-\beta_{p}-1\right)=-F_{S G, 1}(N, p, q)=0 .
$$

We see by (9.2) and (9.5) that

$$
\begin{aligned}
\int_{0}^{T} \int_{\mathbb{R}^{N}}|\nu|^{q} \psi_{R}^{*} d x d t & \geq C^{-q} R^{2-(N-1)(q-1)}\left(\int_{0}^{T} \int_{\mathbb{R}^{N}}\left|\partial_{t} u\right|^{p} \psi_{R} d x d t\right)^{q} \\
& \geq C^{-q} \delta_{1}^{q}\left(I\left[g_{1}\right] \varepsilon\right)^{p q} R^{\left(N-\frac{N-1}{2} p\right) q+2-(N-1)(q-1)} \\
& \geq C^{-q} \delta_{1}^{q}\left(I\left[g_{1}\right] \varepsilon\right)^{p q} R^{\left(N-\frac{N-1}{2} p-\beta_{q}\right) q+N-\frac{N-1}{2} q} \\
& =C^{-q} \delta_{1}^{q}\left(I\left[g_{1}\right] \varepsilon\right)^{p q} R^{\beta_{p}+1}
\end{aligned}
$$

and therefore

$$
\int_{0}^{T} \int_{\mathbb{R}^{N}}|\nu|^{q} \Phi_{\beta, \lambda} \psi_{R}^{*} d x d t \geq C^{-q} \delta_{1}^{q}\left(\varepsilon I\left[g_{1}\right]\right)^{p q}
$$

with $\beta=\beta_{p}+1$ and $\lambda=\lambda_{\beta_{p}+1}$. On the other hand, using Lemma 3.7(ii) with $\beta=\beta_{p}+1$, Lemma 3.8, (9.5) and the condition $F_{S G, 1}(N, p, q)=0$ again, we deduce

$$
\begin{aligned}
\left(\int_{0}^{T} \int_{\mathbb{R}^{N}}|v|^{q} \Phi_{\beta, \lambda} \psi_{R} d x d t\right)^{p q} & \leq\left(\frac{C}{R} \int_{0}^{T} \int_{\mathbb{R}^{N}}\left|\partial_{t} u\right| \Phi_{\beta, \lambda}\left[\psi_{R}\right]^{\frac{1}{p}} d x d t\right)^{p q} \\
& \leq C R^{-\left(N-\frac{N-1}{2} p\right) q}(\log R)^{q(p-1)}\left(\int_{0}^{T} \int_{\mathbb{R}^{N}}\left|\partial_{t} u\right|^{p} \psi_{R}^{*} d x d t\right)^{q} \\
& \leq C R^{-\left(\beta_{p}+1\right)}(\log R)^{q(p-1)} \int_{0}^{T} \int_{\mathbb{R}^{N}}|v|^{q} \psi_{R}^{*} d x d t \\
& \leq C(\log R)^{q(p-1)} \int_{0}^{T} \int_{\mathbb{R}^{N}}|v|^{q} \Phi_{\beta, \lambda} \psi_{R}^{*} d x d t .
\end{aligned}
$$

Lemmas 3.9 and 3.10 with $\delta=\varepsilon^{p q}, p_{1}=p q$ and $p_{2}=q(p-1)+1$ imply $T \leq \exp \left(C \varepsilon^{-p(p q-1)}\right)$.

(The case $F_{S G, 1}(N, p, q)<0=F_{S G, 2}(N, p, q)$ ). Observe that the condition $F_{S G, 2}(N, p, q)=0$ yields

$$
\left(N-\frac{N-1}{2} p-\beta_{q}\right)+\left(N-\frac{N-1}{2} q-\beta_{p}-1\right) p=-F_{S G, 2}(N, p, q)=0 .
$$

We see by (9.3) and (9.4) that

$$
\begin{aligned}
\int_{0}^{T} \int_{\mathbb{R}^{N}}\left|\partial_{t} u\right|^{p} \psi_{R}^{*} d x d t & \geq C^{-p} R^{1-N(p-1)}\left(\int_{0}^{T} \int_{\mathbb{R}^{N}}|v|^{q} \psi_{R} d x d t\right)^{p} \\
& \geq C^{-p} \delta_{2}^{p}\left(I\left[g_{2}\right] \varepsilon\right)^{p q} R^{\left(N-\frac{N-1}{2} q\right) p+1-N(p-1)} \\
& =C^{-p} \delta_{2}^{p}\left(I\left[g_{2}\right] \varepsilon\right)^{p q} R^{\beta_{q}}
\end{aligned}
$$

and therefore

$$
\int_{0}^{T} \int_{\mathbb{R}^{N}}\left|\partial_{t} u\right|^{p} \Phi_{\beta, \lambda} \psi_{R}^{*} d x d t \geq C^{-p} \delta_{2}^{p}\left(I\left[g_{2}\right] \varepsilon\right)^{p q}
$$


with $\beta=\beta_{q}$ and $\lambda=\lambda_{\beta_{q}}$. On the other hand, using Lemma3.7(i) with $\beta=\beta_{q}$, Lemma 3.8, 9.5) and the condition $F_{S G, 2}(N, p, q)=0$, we have

$$
\begin{aligned}
\left(\int_{0}^{T} \int_{\mathbb{R}^{N}}\left|\partial_{t} u\right|^{p} \Phi_{\beta, \lambda} \psi_{R} d x d t\right)^{p q} & \leq\left(\frac{C}{R} \int_{0}^{T} \int_{\mathbb{R}^{N}}|v| \Phi_{\beta+1, \lambda}\left[\psi_{R}^{*}\right]^{\frac{1}{q}} d x d t\right)^{p q} \\
& \leq C R^{-\left(N-\frac{N-1}{2} q\right) p}(\log R)^{p(q-1)}\left(\int_{0}^{T} \int_{\mathbb{R}^{N}}|v|^{q} \psi_{R}^{*} d x d t\right)^{p} \\
& \leq C R^{-\beta_{q}}(\log R)^{p(q-1)} \int_{0}^{T} \int_{\mathbb{R}^{N}}\left|\partial_{t} u\right|^{p} \psi_{R}^{*} d x d t \\
& \leq C(\log R)^{p(q-1)} \int_{0}^{T} \int_{\mathbb{R}^{N}}\left|\partial_{t} u\right|^{p} \Phi_{\beta, \lambda} \psi_{R}^{*} d x d t
\end{aligned}
$$

Lemmas 3.9 and 3.10 with $\delta=\varepsilon^{p q}, p_{1}=p q$ and $p_{2}=p(q-1)+1$ imply $T \leq \exp \left(C \varepsilon^{-q(p q-1)}\right)$.

(The case $\left.F_{S G, 1}(N, p, q)=F_{S G, 2}(N, p, q)=0\right)$. In this case we see from (9.6) and (9.7) that

$$
N-\frac{N-1}{2} p=\beta_{q}, \quad N-\frac{N-1}{2} q=\beta_{p}+1 .
$$

This implies that (9.2) can be rewritten as

$$
\begin{aligned}
\delta_{1}\left(I\left[g_{1}\right] \varepsilon\right)^{p} & \leq R^{-N+\frac{N-1}{2} p} \int_{0}^{T} \int_{\mathbb{R}^{N}}\left|\partial_{t} u\right|^{p} \psi_{R}^{*} d x d t \\
& \leq C \int_{0}^{T} \int_{\mathbb{R}^{N}}\left|\partial_{t} u\right|^{p} \Phi_{\beta, \lambda} \psi_{R}^{*} d x d t
\end{aligned}
$$

with $\beta=\beta_{q}$ and $\lambda=\lambda_{\beta_{q}}$. In view of the above estimate and (9.8), Lemma 3.9 with $w=\left|\partial_{t} u\right|^{p} \Phi_{\beta, \lambda}$ and Lemma 3.10 with $\delta=\varepsilon^{p}, p_{1}=p q$ and $p_{2}=p(q-1)+1$ imply $T \leq \exp \left(C \varepsilon^{-(p q-1)}\right)$. 


\section{References}

[1] R. Agemi, Blow-up of solutions to nonlinear wave equations in two space dimensions, Manuscripta Math. 73 (1991), 153-162.

[2] R. Agemi, Y. Kurokawa, H. Takamura, Critical curve for p-q systems of nonlinear wave equations in three space dimensions, J. Differential Equations 167 (2000), no. 1, 87-133.

[3] R. Beals, R. Wong, "Special functions and orthogonal polynomials," Cambridge Studies in Advanced Mathematics, 153, Cambridge University Press, Cambridge, 2016.

[4] D. Del Santo, V. Georgiev, E. Mitidieri, Global existence of the solutions and formation of singularities for a class of hyperbolic systems, "Geometric Optics and Related Topics" (F. Colombini and N. Lerner Eds.), Progress in Nonlinear Differential Equations and Their Applications, 32, pp.117-140, Birkhäuser Boston, 1997.

[5] D. Del Santo, E. Mitidieri, Blow-up of solutions of a hyperbolic system: the critical case, Differential Equations 34, (1998), 1157-1163.

[6] K. Deng, Nonexistence of global solutions of a nonlinear hyperbolic system, Trans. Amer. Math. Soc. 349 (1997), 1685-1696.

[7] K. Deng, Blow-up of solutions of some nonlinear hyperbolic systems, Rocky Mountain J. Math. 29 (1999), 807-820.

[8] S. Di Pomponio and V. Georgiev, Life-span of subcritical semilinear wave equation, Asymptotic Anal. 28 (2001), 91-114.

[9] M. Escobedo, M.A. Herrero, Boundedness and blow up for a semilinear reaction-diffusion system J. Differential Equations 89 (1991), 176-202.

[10] H. Fujita, On the blowing up of solutions of the Cauchy problem for $u_{t}-\Delta u=u^{1+\alpha}$, J. Fac. Sci. Univ. Tokyo Sect. I 13 (1966). 109-124.

[11] V. Georgiev, H. Lindblad, C.D. Sogge, Weighted Strichartz estimates and global existence for semilinear wave equations, Amer. J. Math. 119 (1997), 1291-1319.

[12] V. Georgiev, H. Takamura, Y. Zhou, The lifespan of solutions to nonlinear systems of a highdimensional wave equation, Nonlinear Anal. 64 (2006), 2215-2250.

[13] R.T. Glassey, Finite-time blow-up for solutions of nonlinear wave equations, Math. Z. 177 (1981), 323-340.

[14] R.T. Glassey, Existence in the large for $\square u=f(u)$ in two space dimensions, Math. Z. 178 (1981), 233-261.

[15] K. Fujiwara, T. Ozawa, Finite time blowup of solutions to the nonlinear Schrödinger equation without gauge invariance, J. Math. Phys. 57 (2016), 082103, 8 pp.

[16] K. Hayakawa, On nonexistence of global solutions of some semilinear parabolic differential equations, Proc. Japan Acad. 49 (1973), 503-505. 
[17] K. Hidano, K. Tsutaya, Global existence and asymptotic behavior of solutions for nonlinear wave equations, Indiana Univ. Math. J. 44 (1995), 1273-1305.

[18] K. Hidano, C. Wang, K. Yokoyama, The Glassey conjecture with radially symmetric data, J. Math. Pures Appl. (9) 98 (2012), 518-541.

[19] K. Hidano, C. Wang, K. Yokoyama, Combined effects of two nonlinearities in lifespan of small solutions to semi-linear wave equations, Math. Ann. 366 (2016), 667-694.

[20] K. Hidano, K. Yokoyama, Life span of small solutions to a system of wave equations, Nonlinear Anal. 139 (2016), 106-130.

[21] M. Ikeda, T. Ogawa, Lifespan of solutions to the damped wave equation with a critical nonlinearity, J. Differential Equations 261 (2016), 1880-1903.

[22] M. Ikeda, M. Sobajima, Life-span of blowup solutions to semilinear wave equation with spacedependent critical damping, arXiv: 1709.04401.

[23] M. Ikeda, M. Sobajima, Life-span of solutions to semilinear wave equation with time-dependent critical damping for specially localized initial data, Math. Ann., to appear.

[24] M. Ikeda, M. Sobajima, Upper bound for lifespan of solutions to certain semilinear parabolic, dispersive and hyperbolic equations via a unified test function method, arXiv: 1710.06780.

[25] M. Ikeda, Y. Wakasugi, Small-data blow-up of $L^{2}$-solution for the nonlinear Schrödinger equation without gauge invariance, Differential Integral Equations 26 (2013), 1275-1285.

[26] F. John, Blow-up of solutions of nonlinear wave equations in three space dimensions, Manuscripta Math. 28 (1979), 235-268.

[27] F. John, Blow-up of solutions for quasi-linear wave equations in three space dimensions, Comm. Pure Appl. Math. 34 (1981), 29-51.

[28] K. Kobayashi, T. Sirao, H. Tanaka, On the growing up problem for semilinear heat equations, J. Math. Soc. Japan 29 (1977), 407-424.

[29] T.Kato, Blow up of solutions of some nonlinear hyperbolic equations, Comm. Pure Appl. Math. 33 (1980), 501-505.

[30] L. V. Kapitanskii, The Cauchy problem for the semilinear wave equation. I, (Russian) Zap. Nauchn. Sem. Leningrad. Otdel. Mat. Inst. Steklov. (LOMI) 163 (1987), Kraev. Zadachi Mat. Fiz. i Smezhn. Vopr. Teor. Funktsii 19, 760-104, 188; translation in J. Soviet Math. 49 (1990), no. 5, 1166-1186.

[31] H. Kubo, M. Ohta, Critical blowup for systems of semilinear wave equations in low space dimensions, J. Math. Anal. Appl. 240 (1999), 340-360.

[32] H. Kubo, K. Kubota, H. Sunagawa, Large time behavior of solutions to semilinear systems of wave equations, Math. Ann. 335 (2006), 435-478.

[33] Y. Kurokawa, The lifespan of radially symmetric solutions to nonlinear systems of odd dimensional wave equations, Nonlinear Anal. 60 (2005), no.7, 1239-1275. 
[34] Y. Kurokawa, H. Takamura, A weighted pointwise estimate for two dimensional wave equations and its applications to nonlinear systems, Tsukuba J. Math. 27 (2003), no.2, 417-448.

[35] Y. Kurokawa, H. Takamura, K. Wakasa, The blow-up and lifespan of solutions to systems of semilinear wave equation with critical exponents in high dimensions, Differential Integral Equations 25 (2012), 363-382.

[36] N.-A. Lai, Y. Zhou, An elementary proof of Strauss conjecture, Journal of Functional Analysis 267 (2014), 1364-1381.

[37] N.-A. Lai, Y. Zhou, The sharp lifespan estimate for semilinear damped wave equation with Fujita critical power in high dimensions, J. Math. Pures Appl., to appear.

[38] T.-Y. Lee, W.-M. Ni, Global existence, large time behavior and life span of solutions of a semilinear parabolic Cauchy problem, Trans. Amer. Math. Soc. 333 (1992), 365-378.

[39] T.T. Li, Y. Zhou, Breakdown of solutions to $\square u+u_{t}=|u|^{1+\alpha}$, Discrete Contin. Dynam. Systems 1 (1995), 503-520.

[40] H. Lindblad, Blow-up for solutions of $\square u=|u|^{p}$ with small initial data, Comm. Partial Differential Equations 15 (1990), 757-821.

[41] H. Lindblad, C.D. Sogge, Long-time existence for small amplitude semilinear wave equations, Amer. J. Math., 118 (1996), 1047-1135.

[42] J. Lin, K. Nishihara, J. Zhai, Critical exponent for the semilinear wave equation with timedependent damping, Discrete Contin. Dyn. Syst. 32 (2012), 4307-4320.

[43] K. Masuda, Blow-up solutions for quasi-linear wave equations in two space dimensions, Lecture Notes in Num. Appl. Anal. 6 (1983), 87-91.

[44] K. Nishihara, Y. Wakasugi, Critical exponent for the Cauchy problem to the weakly coupled damped wave system, Nonlinear Anal. 108 (2014), 249-259.

[45] M.A. Rammaha, Finite-time blow-up for nonlinear wave equations in high dimensions, Comm. Partial Differential Equations 12(1987), 677-700.

[46] M.A. Rammaha, Nonlinear wave equations in high dimensions, Proceeding of the International Conference on Theory and Applications of Differential Equations (Columbus, OH, March 21-25, 1988), Vol.I,II, Ohio University Press, Athens, OH, 1989, 322-326.

[47] J. Schaeffer, The equation $u_{t t}-\Delta u=|u|^{p}$ for the critical value of $p$, Proc. Roy. Soc. Edinburgh 101A (1985), 31-44.

[48] J. Schaeffer, Finite-time blow-up for $u_{t t}-\Delta u=H\left(u_{r}, u_{t}\right)$ in two space dimensions, Comm. Partial Differential Equations 11 (1986), 513-543,

[49] T.C. Sideris, Global behavior of solutions to nonlinear wave equations in three space dimensions, Comm. Partial Differential Equations 8 (1983), 1219-1323,

[50] T.C. Sideris, Nonexistence of global solutions to semilinear wave equations in high dimensions, J. Differential Equations 52 (1984), 378-406. 
[51] W.A. Strauss, Nonlinear scattering theory at low energy, J. Funct. Anal. 41 (1981), 110-133.

[52] S. Sugitani, On nonexistence of global solutions for some nonlinear integral equations, Osaka J. Math. 12 (1975), 45-51.

[53] H. Takamura, Improved Kato's lemma on ordinary differential inequality and its application to semilinear wave equations, Nonlinear Anal. 125 (2015), 227-240.

[54] H. Takamura, K. Wakasa, The sharp upper bound of the lifespan of solutions to critical semilinear wave equations in high simensions, J. Differential Equations 251 (2011), 1157-1171.

[55] N. Tzvetkov, Existence of global solutions to nonlinear massless Dirac system and wave equations with small data, Tsukuba Math. J. 22 (1998), 198-211.

[56] C. Wang, H. Zhou, Almost global existence for semilinear wave equations with mixed nonlinearities in four space dimensions, J. Math. Anal. Appl. 459 (2018), no. 1, 236-246.

[57] W. Xu, Blowup for systems of semilinear wave equations with small initial data, J. Partial Differential Equations 17 (2004), no. 3, 198-206.

[58] B. Yordanov, Q.S. Zhang, Finite time blow up for critical wave equations in high dimensions, J. Funct. Anal. 231 (2006), 361-374.

[59] Y. Zhou, Life span of classical solutions to $u_{t t}-u_{x x}=|u|^{1+\alpha}$, Chin. Ann. Math. Ser.B 13 (1992), 230-243.

[60] Y. Zhou, Blow up of classical solutions to $\square u=|u|^{1+\alpha}$ in three space dimensions, J. Partial Differential Equations 5 (1992), 21-32.

[61] Y. Zhou, Life span of classical solutions to $\square u=|u|^{p}$ in two space dimensions, Chin. Ann. Math. Ser.B 14 (1993), 225-236.

[62] Y. Zhou, Blow up of solutions to the Cauchy problem for nonlinear wave equations, Chin. Ann. Math. Ser.B 22 (2001), 275-280.

[63] Y. Zhou, Blow up of solutions to semilinear wave equations with critical exponent in high dimensions, Chin. Ann. Math. Ser.B 28 (2007), 205-212.

[64] Y. Zhou, W. Han, Blow up for some semilinear wave equations in multi-space dimensions, Comm. Partial Differential Equations 39 (2014), 651-665.

[65] Y. Zhou, W. Han, Life-Span of Solutions to Critical Semilinear Wave Equations, Comm. Partial Differential Equations 39 (2014), 439-451. 